\title{
17. MIOCENE RED CLAYS OF THE SOUTH ATLANTIC: DISSOLUTION FACIES OF CALCAREOUS OOZES AT DEEP SEA DRILLING PROJECT SITES 519 TO 523, LEG $73^{1}$
}

\author{
Anne Marie Karpoff, Centre de Sédimentologie et Géochimie de la Surface, Strasbourg, France
}

\begin{abstract}
In the South Atlantic, at Sites 519 to 523, the dissolution of calcareous oozes ended in the formation of red clays rich in iron and manganese. The early authigenesis of manganese oxides and clays is described in Miocene marly calcareous oozes. The mineralogical and geochemical influences of basaltic basement weathering are shown by the occurrence of palagonite, authigenic clays, and oxides in the basal sediments. The development of red clay facies can be inhibited by local topographic and paleoceanographic changes, as at Site 520.
\end{abstract}

\section{INTRODUCTION}

Sites 519 to 523 are located along a transect on the eastern flank of the Mid-Atlantic Ridge between $25^{\circ}$ and $30^{\circ} \mathrm{S}$ (Fig. 1). One of the objectives of DSDP Leg 73 was to determine the nature and characteristics of paleoceanographic events during the upper Tertiary, particularly the Miocene. The drilled pelagic sequences of Miocene age are mainly marly oozes and clays, and they have been subjected to extensive dissolution that may be related to weak bottom water circulation and/or cooling episodes. These paleoenvironmental changes are manifested by fluctuations in the carbonate compensation depth and consequently (1) the formation of red clay deposits and (2) the occurrences of diatomaceous layers.

\section{SEDIMENTOLOGY}

According to shipboard lithologic studies and smearslide observations, the composite pelagic sections overlying the basement are made up of series of units (Fig. 2).

\section{Site 519}

From bottom to top, the lowest Miocene unit (Unit 3, $45 \mathrm{~m}$ thick) is mainly marly nannofossil ooze with red marl layers overlying basalt. Unit $2(52 \mathrm{~m}$ thick $)$, of soft nannofossil ooze with scarce diatom occurrences, is Pliocene. The youngest unit, Unit $1(54 \mathrm{~m})$ is made up of upper Pliocene and Quaternary calcareous oozes.

\section{Site 520}

In structure, the seafloor at Site $\mathbf{5 2 0}$ is a silled basin that allowed preservation of laminated diatomites and the formation of slumps. The lowermost Miocene deposits of Unit 3 (48 m thick) are clayey nannofossil chalk and bioturbated claystone with some basaltic fragments. Unit 2 (142.5 m thick) includes several sequences of slumps within the Miocene pelagic deposits, which are nannofossil diatom oozes, diatomites, and clayey nannofossil chalk. An iron-rich and volcaniclastic crust

\footnotetext{
${ }^{1}$ Hsü, K. J., LaBrecque, J. L., et al., Init. Repts. DSDP, 73: Washington (U.S. Govt.
} Printing Office).

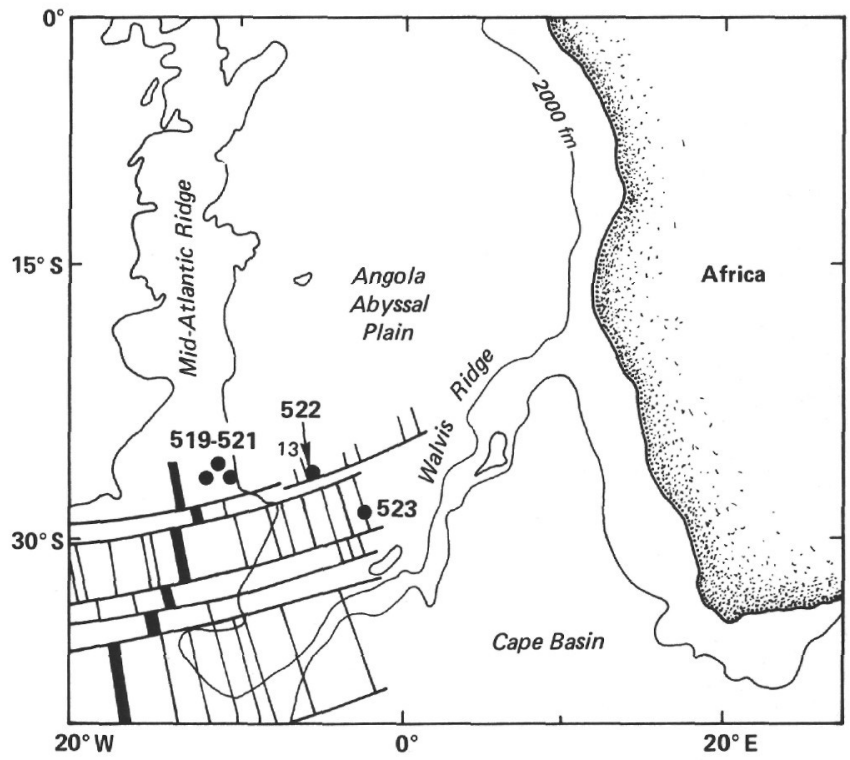

Figure 1. Map of South Atlantic showing Site 519 to 523 locations. Bathymetric contour is $2000 \mathrm{fm}$.

fragment occurs at $305.3 \mathrm{~m}$ sub-bottom within the nannofossil clay. Unit 1 (0-256 m sub-bottom) consists of Pliocene-Quaternary homogeneous nannofossil ooze.

\section{Site 521}

Unit 1 (0-44 $\mathrm{m}$ sub-bottom) is foraminifer nannofossil ooze of Pliocene-Quaternary age. Unit $2(40.2 \mathrm{~m}$ thick) consists of alternating nannofossil clay and marly nannofossil ooze; it is subdivided into Subunits a and $\mathrm{b}$ according to carbonate content. The upper subunit, Sub-unit 2a, which has clay-rich layers, differs from Subunit $2 b$ by the recurrence of foraminifers. The nearness of basaltic basement is shown by the occurrence in places (e.g., Core 7, Section 1) of basalt fragments.

\section{Site 522}

The composite pelagic sequence at Site 522 is remarkably free of slumps and redeposition structures. The 


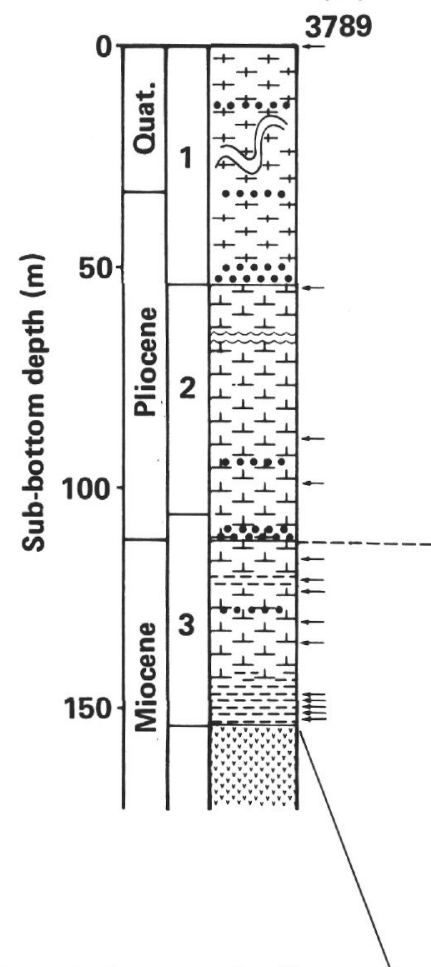

Foraminifer-nannofossil oozes $\stackrel{\perp}{\perp}+\mathcal{L}$ Nannofossil oozes Nannofossil clay and clays E⿰氵气㭋 Diatom clayey layers

\section{Chalk}

Basalt

...... Sands or turbidites

$\Omega$ Slumps

- Study samples

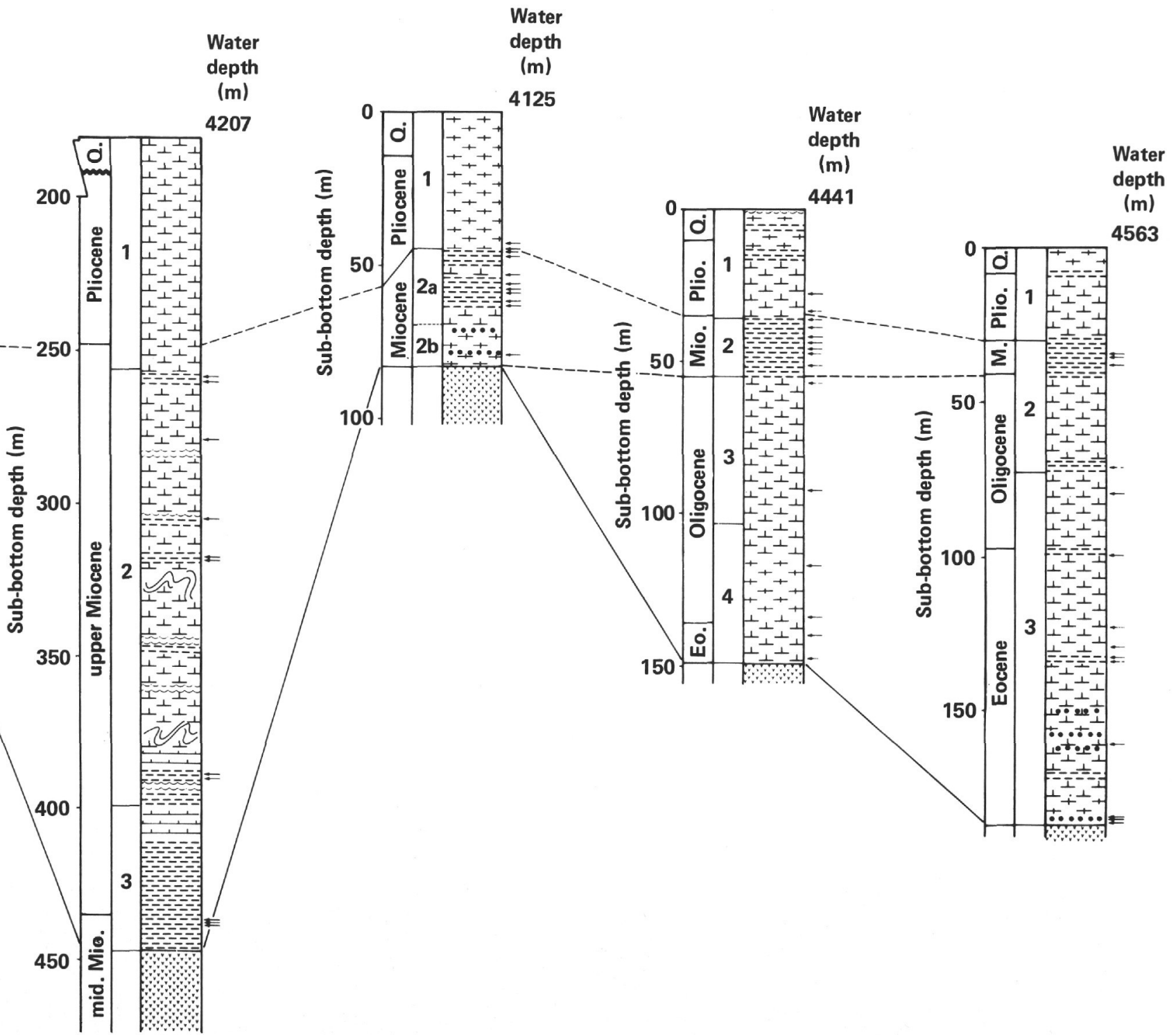

Figure 2. Sediment description and stratigraphy of Sites 519 to 523. Correlation lines are for Miocene deposits (dashed) and basaltic basement (solid). 
lowest units, Units 3 and 4 (about 100 m thick), comprise marly nannofossil oozes and foraminifer nannofossil oozes with small-scale dissolution cycles; the units are of Eocene and Oligocene age. The basal calcareous deposit (Unit 5, Hole 522A) directly above the basalt is rich in volcaniclastic fragments. The Miocene carbonate-poor deposits of reddish brown iron-rich clay that make up Unit 2 (22.3 $\mathrm{m}$ thick) reflect extensive dissolution. The upper unit, Unit 1 (32.8 m thick), which is of Pliocene-Quaternary age, is foraminifer nannofossil ooze and marly ooze.

\section{Site 523}

The deepest eastern ridge site shows the same dominant lithologies as the other sites. The lower part of Unit 3 ( $87.1 \mathrm{~m}$ thick) is pelagic calcareous ooze with volcaniclastic fragments and pebbles of basaltic glass. In the upper part of this unit, several reddish brown clays are intercalated with the nannofossil ooze of EoceneOligocene age. The top of Unit 2 is characterized by dark brown clays and the bottom by marly nannofossil ooze which range in age from Miocene to Oligocene. The upper unit, Unit 1, is still Pliocene-Quaternary nannofossil ooze.

In summary, at all of these ridge flank sites, the Pliocene-Quaternary deposits are calcareous pelagic oozes. The Miocene sediments are marly oozes, nannofossil clays, and red clays. At the eastern sites, Sites 522 and 523 , Oligocene and Eocene nannofossil oozes show small dissolution facies layers and, as at all the sites, occurrences of volcaniclastic fragments in the basal sediments.

The studied samples are mainly from clay-rich and carbonate-poor layers, and their positions are shown in Figure 2 by the arrows next to the sedimentary columns.

\section{MINERALOGY}

\section{Methods}

The mineralogical composition of the sediments was determined by X-ray diffraction techniques. X-ray diffraction charts were obtained from non-oriented powders by using bulk material under the following conditions: $\mathrm{CuK} \alpha$ radiation, Ni filter, $98 \mathrm{kV} / 18 \mathrm{~mA} ; 0.1$ to $1^{\circ}$ slits, $1 \% / \mathrm{min}$. speed. The clay minerals in the $<2 \mu \mathrm{m}$ fractions were identified according to the methods of the Institut de Geologie de Strasbourg (see Mise au point collective, 1975) on three types of oriented aggregates: (1) untreated, (2) ethylene-glycol treated, and (3) heated.

Some clay fractions were also studied under a Phillips EM300 transmission electron microscope (TEM) by using the method of Trauth et al. (1977).

\section{Results}

The data obtained on the bulk sediments and clay fractions for each site are presented in Figures 3 to 7. The figures also show the composition of the coarse fraction $(>63 \mu \mathrm{m})$.

\section{Bulk Sediment Mineralogy}

\section{Site 519}

Calcite prevails, with scarce quartz and feldspars (Fig. 3). In the lowest Miocene deposits (those overlying the basement), the clays become more abundant. In the coarse fraction, calcareous organisms dominate. Black micronodules are abundant at the top of Miocene Unit 3 ; at the bottom they are associated with palagonite, scarce volcanic glass, and quartz. Black manganese oxide patches on foraminifers from the upper Miocene calcareous deposits correlate with the gradual formation of the coarse micronodules.

\section{Site 520}

The progressive variations in the bulk mineralogy at this site (Fig. 4) correspond to changes in $\mathrm{CaCO}_{3}$ content, which ranges downward from $60 \%$ to $10 \%$. Decreases in $\mathrm{CaCO}_{3}$ correspond to increases in quartz, feldspars, clays, and organic silica in the diatomaceous layers. In the coarse fraction, palagonite occurs in Unit 3 , which overlies the basalt; it is associated with abundant micronodules of manganese. The manganese micronodules are also present in the calcareous sequence; they are scarce in the diatom oozes.

Photomicrographs of the micronodule-rich coarse fraction are shown in Plate 1. The micronodules form when manganese oxides crystallize in foraminifer tests and are deposited in successive thin layers (Plate 1, figs. 3 and 4). The manganese oxides are remarkably well crystallized in this siliceous environment. Such an association (of preserved siliceous organisms and crystallized manganese oxides) in the Miocene pelagic sediments can be related to the combined effects of cooling and changes in the topography of the site.

There is a crust fragment (see Plate 6, figs. 2 and 4) at the transition between the calcareous oozes and nannofossil clay $(520-15-2,31 \mathrm{~cm})$. The bottom of this indurated sedimentary fragment is composed of volcanic minerals, including pyroxenes, plagioclases, palagonite, and red iron oxides, and it has a breccia-like structure; the successive iron-oxide layers on the top of the fragment comprise quartz and clays. These layers have colloform structures like those of the deep-sea metalliferous nodules (Sorem and Fewkes, 1977).

\section{Site 521}

The bulk mineralogical composition of the pelagic sequences at this site shows again the strong relation between low calcite contents and increasing contents of quartz, feldspars, and clays (Fig. 5). Near the basaltic basement the coarse fraction of the clayey basal sediment is enriched in micronodules and palagonite grains.

Microphotographs (Plate 2) show volcaniclastic particles in the coarse fraction of the calcareous ooze of Pliocene age, establishing the sporadic but frequent influence of the volcanic activity of the ridge on the pelagic sedimentation. The alteration of these volcaniclastic fragments is a source of authigenic minerals, such as sil- 


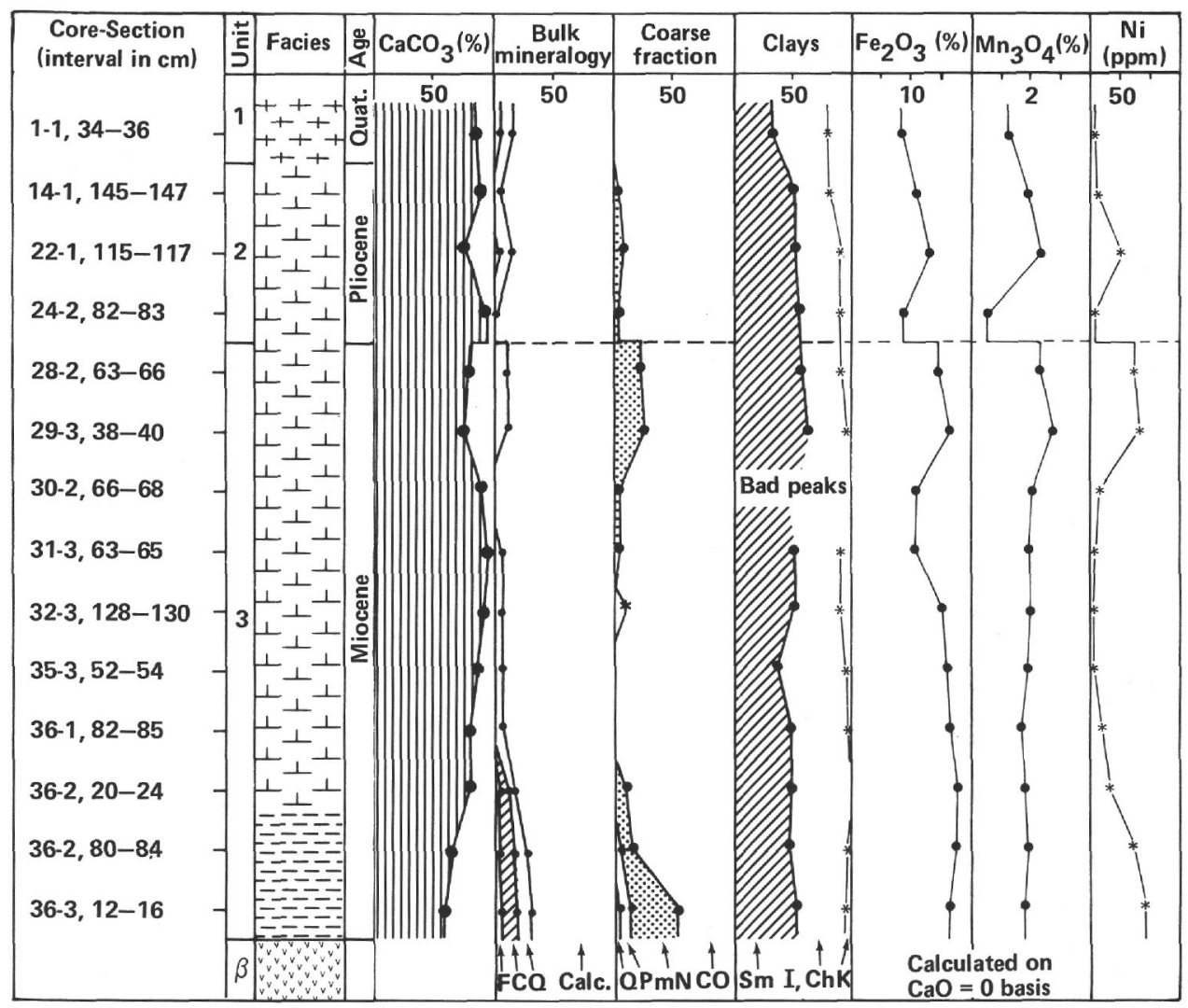

Figure 3. Summary of sedimentological, mineralogical, and geochemical data for Site 519. F = Feldspars, $\mathrm{C}=$ calcite, $\mathrm{Q}=$ quartz, $\mathrm{Calc}=$ calcite, $\mathrm{P}=$ palagonite, $\mathrm{mN}=$ micronodules, $\mathrm{CO}=$ calcareous organisms, $\mathrm{Sm}=$ smectites, $\mathrm{I}, \mathrm{Ch}=$ illite and chlorite, $\mathrm{K}=$ kaolinite. $\beta=$ basalt.

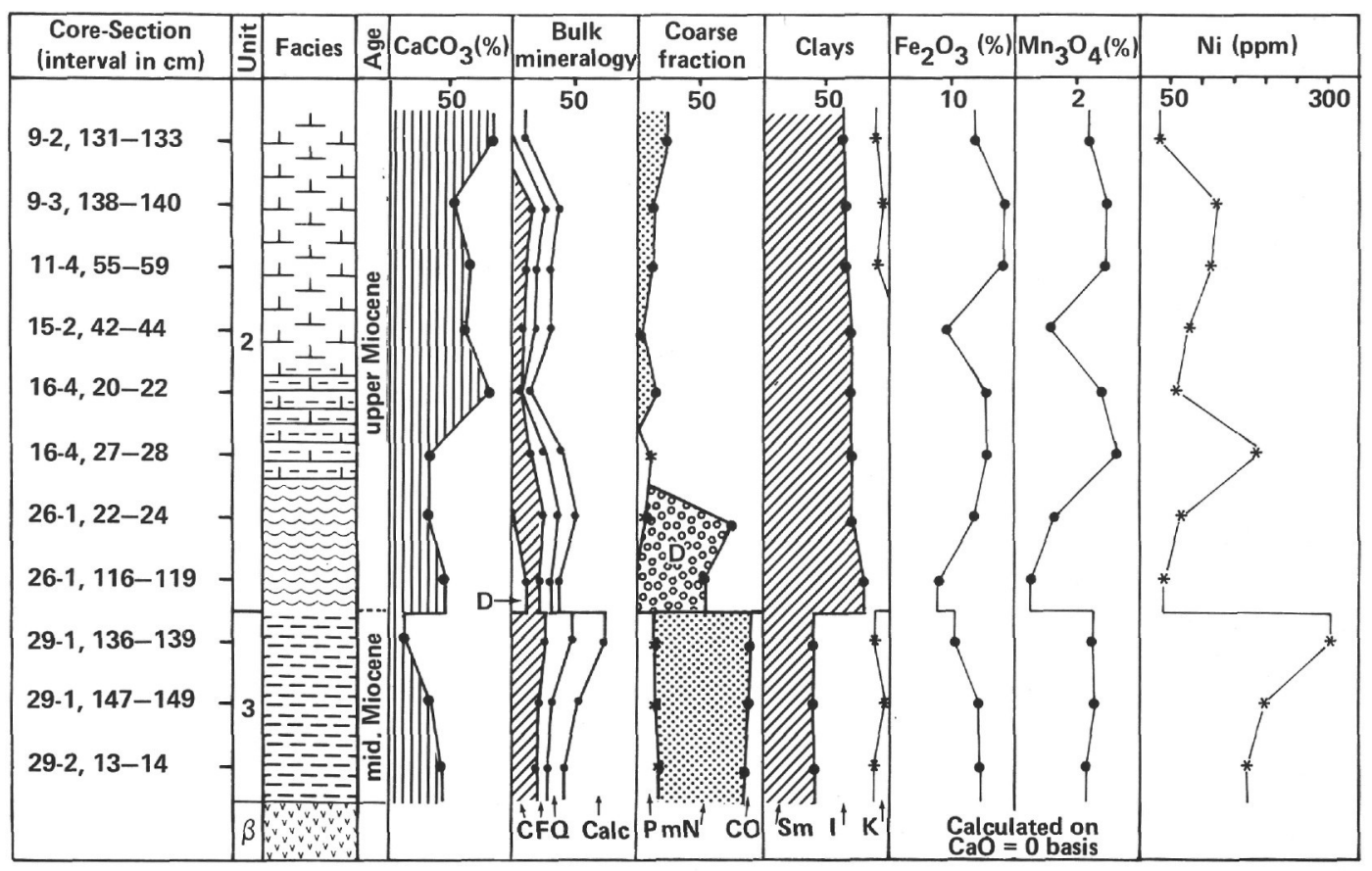

Figure 4. Summary of the sedimentological, mineralogical and geochemical data for Site 520 deposits. Symbols as in Figure 3; $\mathrm{D}=$ diatoms. 


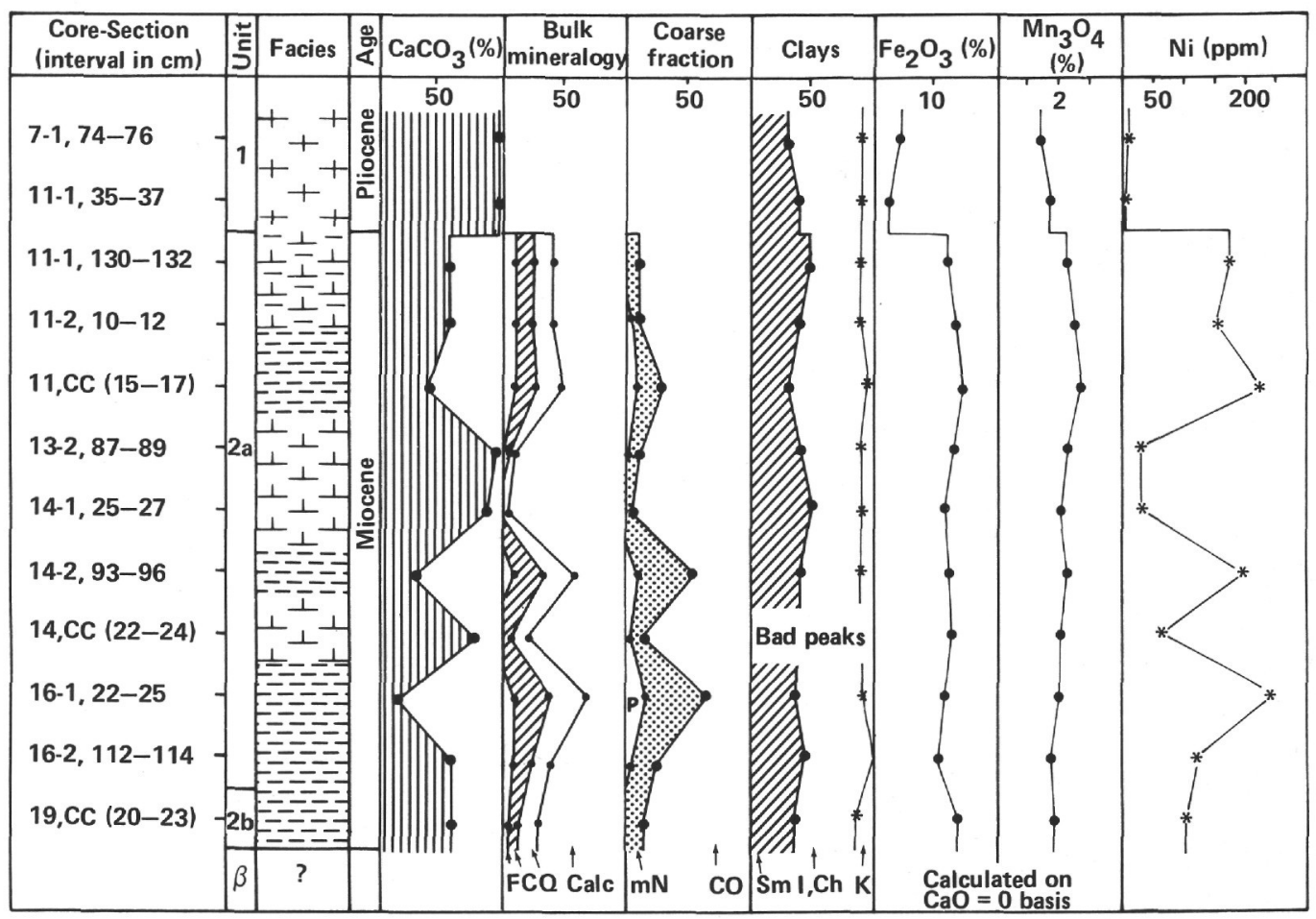

Figure 5. Summary of the sedimentological, mineralogical and geochemical data on Site 521 deposits. Symbols as in Figure 3.

icates and oxides, which can be mixed with the detrital components and promote their transformation during dissolution processes or the early diagenesis of the host pelagic deposits.

\section{Site 522}

The Miocene sediment samples contain an abundant clay fraction, quartz, and feldspars (Fig. 6). The small coarse fraction is rich in micronodules. At the base of this hole, above the basaltic rocks, palagonite appears in the coarse fraction with micronodules.

\section{Site 523}

The Miocene sediments have the same mineralogical characteristics, such as abundance of clays and detrital components, and authigenic micronodules, as at previous sites. These components decrease in the Oligocene and Eocene calcareous sequences (Fig. 7), but micronodules stay present into the basal sediments.

\section{Mineralogy of Altered Basement}

In order to compare the mineralogical composition of the basal sediments and the altered seafloor, X-ray analyses and scanning electron microscope (SEM) observations were made on an altered volcanic breccia from Site 519 (Sample 519A-8-4, 139-144 cm (Piece 8); Plate 3 ). The association of zeolites and smectites with volcanic glass and palagonite is well known and is similar to associations described by Sheppard and Gude (1969) and Honnorez (1978). The smectites have the same shape as volcanogenic authigenic clays described by Borst and Keller (1969), Khoury and Eberl (1979), and Karpoff et al. (1980). Manganese oxides fill up the small cracks in the palagonite. The breccia contains small rounded granules of dark volcanic glass, with a fine skin of clays before the surrounding rim of yellow palagonite. The glassy granules are cemented by intergranular zeolites such as radial clusters of phillipsite.

All these mineralogical parageneses except the phillipsite occur in the sediments overlying the basalt. Phillipsite seems to be very unstable in such calcareous deposits in the South Atlantic, as suggested by Kastner and Stonecipher (1978).

In summary, in the bulk sediments of all the ridge sites, the feldspars are interpreted as continental detritus and/or volcanic residue. Quartz is obviously a continental detrital mineral in the South Atlantic; no other source of quartz has yet been determined. The ratio of feldspar to quartz (two scarce detrital components) appears a little higher in the Miocene deposits than in the other pelagic sequences. The siliceous clay and iron-rich crust found at Site 520 is quite similar to the clay concretion described at Site 464 in the Pacific (Karpoff et al., 1981) and is an indication that silica was contributed from some source during the early diagenetic evolution of the pelagic sediments in the South Atlantic, particularly siliceous calcareous biogenic oozes deposited in restricted areas such as silled basins.

The contribution of basalt weathering is manifested by the increase in smectites, manganese oxides, palagonite, and glassy clasts in the basal sediments and by the sporadic appearance of these minerals in the pelagic section. Meanwhile the specific dissolution processes taking place in the pelagic oozes are manifested by the acquisition of the reddish brown to dark brown color related to an increasing content of diffuse iron-oxyhy- 


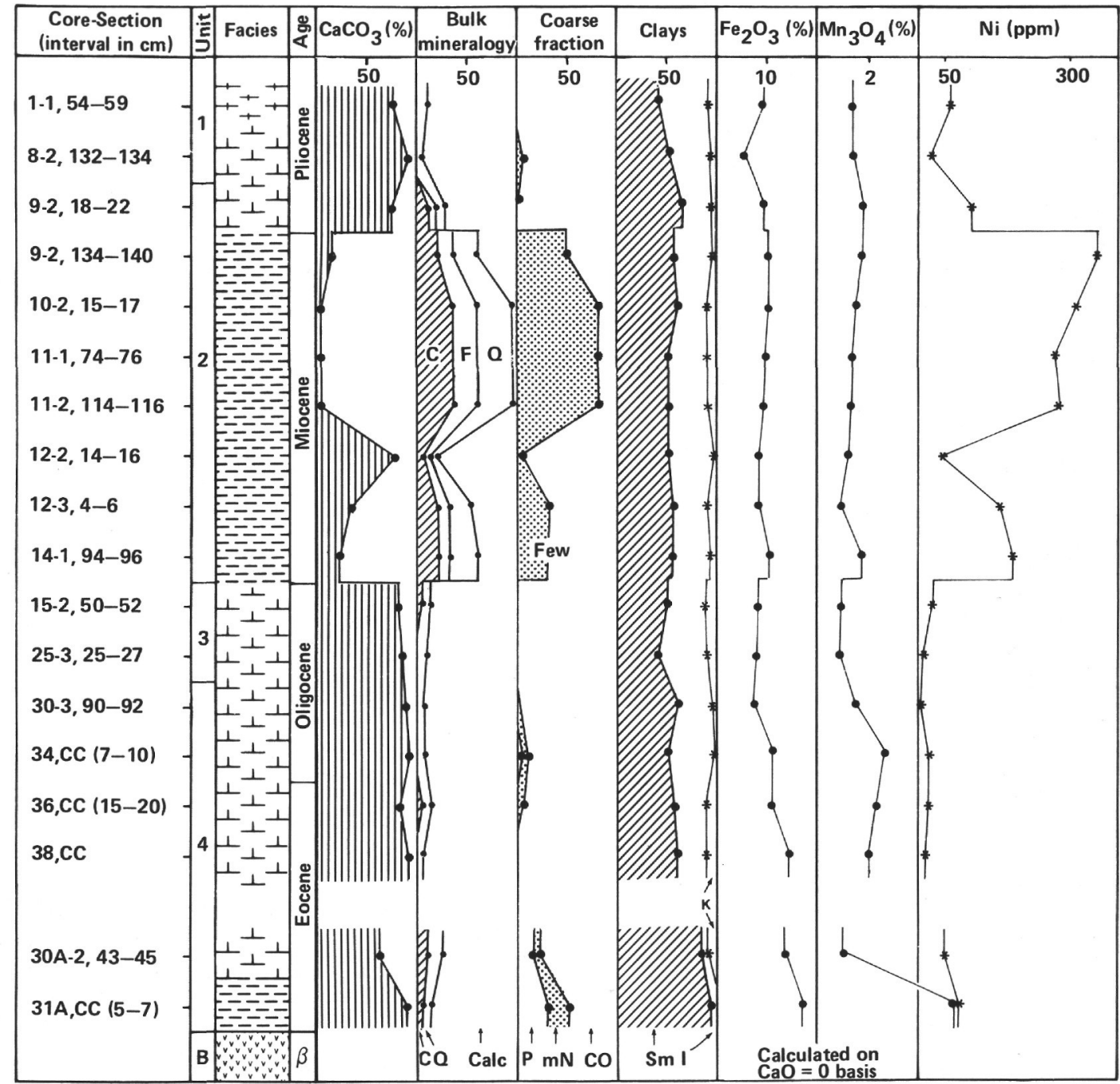

Figure 6. Summary of the sedimentological, mineralogical and geochemical data on Site 522 deposits. Symbols as in Figure 3.

droxides in the clay fraction; the manganiferous micronodules are prevalent in the coarse fraction.

\section{Clay Fraction Composition}

For all the sites, the clay fraction composition is mainly homogeneous, with prevalent smectites, rare or no kaolinite and illite, and low contents of mixed-layer minerals (Figs. 3-7). The proportion of smectites in the clay fraction ranges from 40 to $50 \%$ in the Miocene oozes at Site 519 to $90 \%$ in the Eocene deposits at Site 523. The details of the variations in contents and X-ray diffraction patterns are as follows.

At Site 519,40 to $50 \%$ of the fine fraction of the basal Miocene marl is made up of smectites, with a slight increase near the basalt; the reflection peak of smectites is at $15 \AA$ in the Pliocene deposits, at $14 \AA$ near the top of Miocene sequence, and at $12 \AA$ in the basal sediments.

At Site 520 , the smectite contents $(40-70 \%)$ in the upper Miocene diatomites are higher than in the middle Miocene marly calcareous layers; the prominent peak varies between $15 \AA$ and $14 \AA$ in the upper deposits and shifts to $12 \AA$ in the basal sediments.
At Sites 521, 522, and 523, the Miocene clays are 40 to $50 \%$ smectites, the Oligocene clay 50 to $60 \%$, the basal Eocene deposits $90 \%$ smectites. The X-ray diagrams show a mixture of $14 \AA$ and prevalent $15 \AA$ smectites in the Miocene clay sequence and $12 \AA$ and 15 $\AA$ peaks in the basal sediments; some carbonate-rich samples contain a minor clay fraction with very weak peaks.

The changes in the X-ray characteristics of the smectites were noticed by Hoffert (1980) for the smectites from calcareous oozes (14 $\AA$ ) and the authigenic clays from deposits near the East Pacific Rise (12-13 $\AA$ ).

\section{Scanning Electron Microscope Studies of the Clay Fraction}

The morphologies of the different clay fractions were studied by transmission electron microscope. The nature of the particles was examined by using electron diffraction diagrams.

The smectites from the basal sediments (Plate 4) have two types of morphologies. The smaller particles have hairy outlines and are often like aggregates of short bent laths (Plate 4, figs. 1, 3,4). These forms suggest authi- 


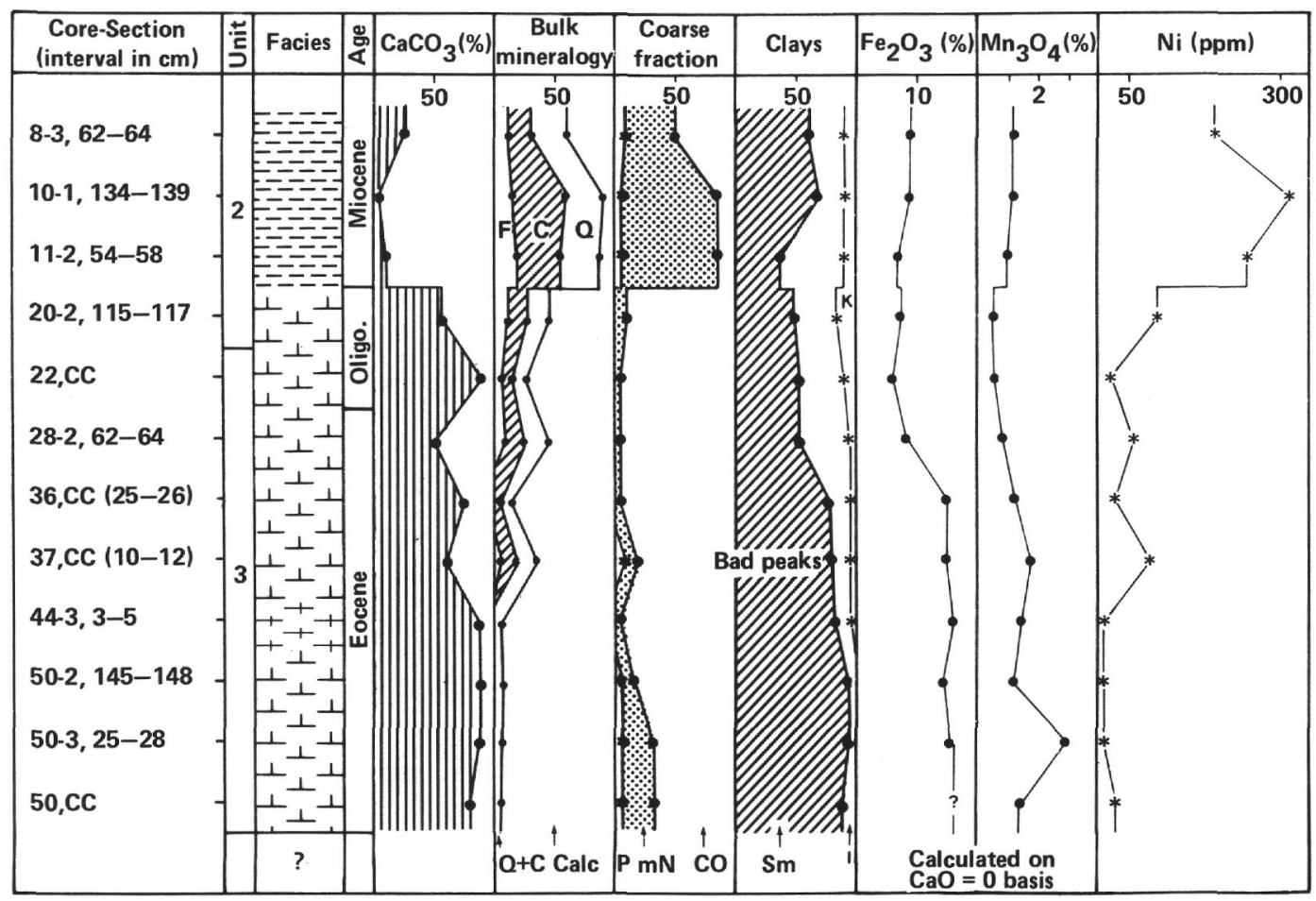

Figure 7. Summary of the sedimentological, mineralogical and geochemical data on Site $\mathbf{5 2 3}$ deposits. Symbols as in Figure 3.

genic iron smectites. The large particles have the curled edges typical of well crystallized detrital smectites (Plate 4, fig. 2) or show lath-shaped edges and light curls, like smectites from the dissolution facies of calcareous oozes (see Hoffert, 1980). These morphologies are identical in the Miocene (Site 519) and Eocene (Hole 522B) basal sediments.

In the reddish clay sequences of Miocene age (Sites 521 and 522), with little seafloor basaltic contribution, the smectite particles have essentially the morphologies of transformed clays, with fine lath overgrowths (Plate $5)$. The iron oxides generally form aggregates or small granules (Plate 5, fig. 5) like those described by Schwertmann and Taylor $(1977,1979)$. In some clay fractions (Plate 5, figs. 6 and 7), the small flakes are similar to manganese-iron oxides (Chukhrov et al., 1980), and the fine veils have the same shape as allophanes (Violante and Tait, 1978; Parfitt and Hemni, 1980).

The clay fraction from the marly nannofossil ooze below the iron crust at Site 520 (Plate 6) is made up of detrital clay and a few transformed particles of smectites (Plate 6, figs. 3-5). The particles are similar to those from dissolution facies of calcareous oozes, with some hairy overgrowths. Such a morphology suggests a comparison with the hisingerite, a poorly crystalline form of nontronite described by Kohyama and Sudo (1975).

\section{GEOCHEMISTRY}

\section{Methods}

The geochemical data were derived from the same set of samples and clay fractions. Major element analyses were performed by following the method described by Besnus and Rouault (1973), which uses arc spectrometry and an ARL quantometer. The method consists of melting the sample mixed with lithium tetraborate and introducing the melt into a glycolated solvent. Trace elements were determined by using an inductively coupled plasma technique (ICP-35000 C-ARL). Na and K were determined by emission spectrometry. Relative precision is $\pm 2 \%$ for the major elements and $\pm 10 \%$ for the trace elements.

\section{Results}

The bulk chemical data are presented in Tables 1 to 15 , which present the major element chemistry, the major element chemistry recalculated on a $\mathrm{CaCO}_{3}$-free basis (correction for $\mathrm{CaO}=0 \%$ and equivalent $\mathrm{CO}_{2}$ from total ignition at $1000^{\circ} \mathrm{C}$ ), and trace element chemistry.

For all the sites, the variations of $\mathrm{Fe}_{2} \mathrm{O}_{3}$ and $\mathrm{Mn}_{3} \mathrm{O}_{4}$ contents were calculated on a $\mathrm{CaO}$-free basis. $\mathrm{Ni}$ contents are reported along with the schematic sedimentary columns in Figures 3 to 7.

\section{Bulk Sediments}

Variations in the major elements, which reflect variations in mineralogical composition, accompany changes in carbonate content. Simultaneous increases in $\mathrm{Al}, \mathrm{Mg}$, $\mathrm{Ti}$, and $\mathrm{K}$ contents are associated with increases in clays, and the occurrence of oxides is revealed by high $\mathrm{Mn}$ values. Iron is located in both phases, which are closely associated. The relative variation of oxides and clays is shown by the iron-to-manganese ratio (Tables 2, 5, 8, 11 , and 14). 
Table 1. Major element chemistry (wt.\%) of sedimentary deposits, Site 519.

\begin{tabular}{|c|c|c|c|c|c|c|c|c|c|c|c|c|}
\hline $\begin{array}{l}\text { Core-Section } \\
\text { (interval in cm) }\end{array}$ & $\mathrm{SiO}_{2}$ & $\mathrm{Al}_{2} \mathrm{O}_{3}$ & $\mathrm{MgO}$ & $\mathrm{CaO}$ & $\mathrm{Fe}_{2} \mathrm{O}_{3}$ & $\mathrm{Mn}_{3} \mathrm{O}_{4}$ & $\mathrm{TiO}_{2}$ & $\mathrm{BaO}$ & $\mathrm{Na}_{2} \mathrm{O}$ & $\mathrm{K}_{2} \mathrm{O}$ & $\operatorname{Ign}^{a}$ & Total \\
\hline $1-1,34-36$ & 5.8 & 1.8 & 0.67 & 49.0 & 1.0 & 0.159 & 0.12 & 0.05 & 0.11 & $<0.05$ & 41.32 & 100.19 \\
\hline $14-1,145-147$ & 3.6 & 1.2 & 0.49 & 51.3 & 0.9 & 0.155 & 0.09 & $<0.01$ & 0.10 & $<0.05$ & 41.99 & 99.96 \\
\hline $22-1,115-117$ & 27.4 & 1.0 & 0.61 & 37.0 & 0.6 & 0.067 & 0.05 & 0.05 & 0.46 & 0.05 & 51.70 & 99.08 \\
\hline $16-1,101-102$ & 10.2 & 3.3 & 0.97 & 43.0 & 2.8 & 0.512 & 0.19 & 0.02 & 0.41 & $<0.05$ & 37.04 & 98.57 \\
\hline $24-2,82-83$ & 2.8 & 0.8 & 0.45 & 52.9 & 0.5 & 0.032 & 0.07 & $<0.01$ & 0.12 & $<0.05$ & 42.43 & 100.25 \\
\hline $28-2,63-66$ & 10.2 & 3.2 & 1.01 & 44.8 & 2.9 & 0.487 & 0.17 & 0.01 & 0.39 & $<0.05$ & 37.18 & 100.48 \\
\hline $29-1,95-96$ & 3.0 & 0.9 & 0.43 & 51.2 & 2.1 & 0.162 & 0.14 & $<0.01$ & $<0.05$ & $<0.05$ & 42.19 & 100.38 \\
\hline $29-3,38-40$ & 10.6 & 3.4 & 1.00 & 43.5 & 3.7 & 0.621 & 0.19 & 0.02 & 0.35 & $<0.05$ & 36.65 & 100.43 \\
\hline $30-2,66-68$ & 3.3 & 1.0 & 0.47 & 51.9 & 0.9 & 0.152 & 0.07 & $<0.01$ & 0.12 & $<0.05$ & 42.18 & 100.02 \\
\hline $31-3,63-65$ & 2.0 & 0.7 & 0.42 & 53.5 & 0.5 & 0.098 & 0.06 & $<0.01$ & 0.13 & $<0.05$ & 42.97 & 100.43 \\
\hline $31-3,83-85$ & 2.3 & 0.8 & 0.42 & 52.9 & 0.8 & 0.107 & 0.04 & $<0.01$ & 0.06 & $<0.05$ & 42.85 & 100.41 \\
\hline $32-3,128-130$ & 2.4 & 0.9 & 0.45 & 52.4 & 1.0 & 0.134 & 0.07 & $<0.01$ & 0.11 & $<0.05$ & 42.70 & 100.25 \\
\hline $34-3,50-52$ & 3.3 & 1.0 & 0.45 & 50.7 & 1.3 & 0.152 & 0.06 & $<0.01$ & 0.05 & $<0.05$ & 41.99 & 99.15 \\
\hline $35-3,52-54$ & 4.8 & 1.7 & 0.61 & 50.0 & 1.8 & 0.217 & 0.12 & $<0.01$ & 0.15 & $<0.05$ & 40.95 & 100.60 \\
\hline $36-1,82-85$ & 7.5 & 2.7 & 0.80 & 46.2 & 2.8 & 0.275 & 0.17 & $<0.01$ & 0.33 & $<0.05$ & 38.68 & 99.66 \\
\hline $36-1,134-138$ & 7.0 & 2.1 & 0.70 & 47.1 & 3.2 & 0.388 & 0.11 & $<0.01$ & $<0.05$ & $<0.05$ & 39.27 & 100.14 \\
\hline $36-2,20-24$ & 9.3 & 3.2 & 0.95 & 45.0 & 3.7 & 0.387 & 0.19 & $<0.01$ & 0.34 & $<0.05$ & 37.52 & 100.72 \\
\hline $36-2,80-84$ & 15.2 & 5.0 & 1.39 & 36.6 & 5.8 & 0.654 & 0.32 & 0.02 & 0.81 & 0.10 & 32.61 & 98.65 \\
\hline $36-3,12-16$ & 19.3 & 6.4 & 1.66 & 33.6 & 6.7 & 0.740 & 0.36 & 0.02 & 1.19 & 0.29 & 29.94 & 100.40 \\
\hline $37, \mathrm{CC}^{\mathrm{b}}$ & 49.3 & 13.8 & 8.13 & 11.6 & 9.6 & 0.163 & 1.15 & $<0.01$ & 2.44 & 0.11 & 0.36 & 96.62 \\
\hline
\end{tabular}

Note: Total iron is calculated as $\mathrm{Fe}_{2} \mathrm{O}_{3}$ and total manganese as $\mathrm{Mn}_{3} \mathrm{O}_{4}$.

a Ign $=$ ignition at $1000^{\circ} \mathrm{C}$

b Volcanic glass.

Table 2. Major element chemistry (wt.\%) of sedimentary deposits, Site 519, recalculated on CaO-free basis.

\begin{tabular}{lccccccccccccc}
\hline $\begin{array}{c}\text { Core-Section } \\
\text { (interval in cm) }\end{array}$ & $\mathrm{SiO}_{2}$ & $\mathrm{Al}_{2} \mathrm{O}_{3}$ & $\mathrm{MgO}$ & $\mathrm{Fe}_{2} \mathrm{O}_{3}$ & $\mathrm{Mn}_{3} \mathrm{O}_{4}$ & $\mathrm{TiO}_{2}$ & $\mathrm{BaO}$ & $\mathrm{Na}_{2} \mathrm{O}$ & $\mathrm{K}_{2} \mathrm{O}$ & $\mathrm{Ign}^{\mathrm{a}}$ & $\frac{\mathrm{SiO}_{2}}{\mathrm{Al}_{2} \mathrm{O}_{3}}$ & $\frac{\mathrm{Fe}_{2} \mathrm{O}_{3}}{\mathrm{Mn}_{3} \mathrm{O}_{4}}$ & $\mathrm{CaCO}_{3} \mathrm{~b}$ \\
\hline $1-1,34-36$ & 46.11 & 14.31 & 5.33 & 7.95 & 1.26 & 0.95 & 0.40 & 0.87 & 0.40 & 22.42 & 3.22 & 6.30 & 87.5 \\
$14-1,145-147$ & 43.54 & 14.51 & 5.81 & 10.89 & 1.87 & 1.09 & 0.12 & 1.21 & 0.60 & 20.35 & 3.00 & 5.82 & 91.6 \\
$16-1,101-102$ & 83.24 & 3.04 & 1.85 & 1.82 & 0.20 & 0.15 & 0.15 & 1.40 & 0.15 & 7.99 & 27.4 & 9.11 & 66.1 \\
$22-1,115-117$ & 46.99 & 15.20 & 4.47 & 12.90 & 2.36 & 0.88 & 0.09 & 1.89 & 0.23 & 14.99 & 3.09 & 5.46 & 76.8 \\
$24-2,82-83$ & 49.14 & 14.04 & 7.90 & 8.78 & 0.56 & 1.23 & 0.18 & 2.11 & 0.88 & 15.19 & 3.50 & 15.67 & 94.4 \\
$28-2,63-66$ & 50.01 & 15.69 & 4.95 & 14.22 & 2.39 & 0.83 & 0.05 & 1.91 & 0.25 & 9.71 & 3.18 & 5.95 & 80.0 \\
$29-1,95-96$ & 34.08 & 10.22 & 4.88 & 23.85 & 1.84 & 1.59 & 0.11 & 0.57 & 0.57 & 22.28 & 3.33 & 12.96 & 91.4 \\
$29-3,38-40$ & 47.78 & 15.04 & 4.42 & 16.37 & 2.74 & 0.84 & 0.09 & 1.55 & 0.22 & 10.93 & 3.17 & 5.97 & 77.6 \\
$30-2.66-68$ & 44.28 & 13.42 & 6.31 & 10.74 & 2.04 & 0.94 & 0.13 & 1.61 & 0.67 & 19.86 & 3.30 & 5.26 & 92.6 \\
$31-3,63-65$ & 40.80 & 14.28 & 8.57 & 10.20 & 2.00 & 1.22 & 0.20 & 2.65 & 1.02 & 19.06 & 2.85 & 5.10 & 95.5 \\
$31-3,83-85$ & 39.16 & 13.62 & 7.15 & 13.62 & 1.82 & 0.68 & 0.17 & 1.02 & 0.85 & 21.89 & 2.87 & 7.48 & 94.4 \\
$32-3,128-130$ & 36.08 & 13.53 & 6.76 & 15.03 & 2.01 & 1.05 & 0.15 & 1.65 & 0.75 & 22.98 & 2.66 & 7.47 & 93.5 \\
$34-3,50-52$ & 38.70 & 11.73 & 5.28 & 15.25 & 1.78 & 0.70 & 0.12 & 0.59 & 0.59 & 25.27 & 3.30 & 8.56 & 90.5 \\
$35-3,52-54$ & 43.16 & 15.29 & 5.48 & 16.19 & 1.95 & 1.08 & 0.09 & 1.35 & 0.45 & 14.96 & 2.82 & 8.30 & 89.3 \\
$36-1,82-85$ & 44.08 & 15.87 & 4.70 & 16.46 & 1.62 & 1.00 & 0.06 & 1.94 & 0.29 & 13.99 & 2.77 & 10.16 & 82.5 \\
$36-1,134-138$ & 44.11 & 13.23 & 4.41 & 20.16 & 2.44 & 0.69 & 0.06 & 0.32 & 0.32 & 14.26 & 3.33 & 8.26 & 84.1 \\
$36-2,20-24$ & 45.84 & 15.77 & 4.68 & 18.24 & 1.91 & 0.94 & 0.05 & 1.68 & 0.25 & 10.66 & 2.90 & 9.55 & 80.3 \\
$36-2,80-84$ & 45.86 & 15.08 & 4.19 & 17.50 & 1.97 & 0.97 & 0.06 & 2.44 & 0.30 & 11.62 & 3.04 & 8.88 & 65.3 \\
$36-3,12-16$ & 48.01 & 15.92 & 4.13 & 16.67 & 1.84 & 0.90 & 0.05 & 2.96 & 0.72 & 8.81 & 3.01 & 9.06 & 60.0 \\
\hline
\end{tabular}

a Ign = ignition at $1000^{\circ} \mathrm{C}$.
b Calculated from total $\mathrm{CaO}(\%)$.

Table 3. Trace element chemistry (ppm) of sedimentary deposits, Site 519.

\begin{tabular}{lrrrrrrrrrr}
\hline $\begin{array}{c}\text { Core-Section } \\
\text { (interval in cm) }\end{array}$ & $\mathrm{Sr}$ & $\mathrm{Ba}$ & $\mathrm{V}$ & $\mathrm{Ni}$ & $\mathrm{Co}$ & $\mathrm{Cr}$ & $\mathrm{B}$ & $\mathrm{Zn}$ & $\mathrm{Cu}$ & $\mathrm{Pb}$ \\
\hline $1-1,34-36$ & 1964 & 632 & 16 & 6 & $<2$ & $<5$ & $<5$ & $<2$ & 48 & $<2$ \\
$14-1,145-147$ & 2087 & 93 & 5 & 12 & $<2$ & $<5$ & $<5$ & $<2$ & 26 & $<2$ \\
$22-1,115-117$ & 923 & 415 & 10 & 45 & 25 & 12 & - & 30 & 26 & - \\
$16-1,101-102$ & 1820 & 235 & 51 & 55 & 31 & $<5$ & $<5$ & 9 & 89 & 19 \\
$24-2,82-83$ & 1704 & 64 & 9 & 3 & $<2$ & $<5$ & $<5$ & $<2$ & 22 & $<2$ \\
$28-2,63-66$ & 1420 & 147 & 38 & 72 & 33 & 11 & 62 & 63 & 80 & 17 \\
$29-1,95-96$ & 1474 & 54 & 18 & 41 & 29 & 10 & - & 18 & 16 & - \\
$29-3,38-40$ & 1846 & 188 & 73 & 82 & 43 & 21 & 60 & 21 & 100 & 26 \\
$30-2,66-68$ & 1899 & 96 & 22 & 14 & $<2$ & 5 & 26 & $<2$ & 16 & 11 \\
$31-3,63-65$ & 1296 & 75 & $<5$ & 7 & $<2$ & $<5$ & $<5$ & $<2$ & 8 & 12 \\
$31-3,83-85$ & 928 & 70 & 17 & 37 & 29 & 7 & - & 18 & 12 & - \\
$32-3,128-130$ & 1772 & 73 & $<5$ & 6 & $<2$ & $<5$ & $<5$ & $<2$ & 11 & 8 \\
$34-3,50-52$ & 1485 & 60 & 29 & 37 & 30 & 7 & - & 24 & 14 & - \\
$35-3,52-54$ & 2034 & 64 & 24 & 7 & $<2$ & $<5$ & 8 & $<2$ & 30 & $<2$ \\
$36-1,82-85$ & 1815 & 62 & 50 & 21 & 6 & $<5$ & 5 & 5 & 63 & 19 \\
$36-1,134-138$ & 1451 & 62 & 62 & 49 & 36 & 10 & - & 64 & 33 & - \\
$36-2,20-24$ & 1935 & 87 & 70 & 32 & 13 & 5 & 61 & 3 & 61 & 16 \\
$36-2,80-84$ & 1317 & 151 & 117 & 70 & 38 & 7 & 93 & 46 & 108 & 39 \\
$36-3,12-16$ & 1324 & 181 & 116 & 89 & 45 & 19 & 110 & 53 & 103 & 64 \\
$37, C^{\text {a }}$ & 115 & 8 & 235 & 156 & 58 & 396 & - & 78 & 50 & - \\
\hline
\end{tabular}

Note: Dashes $=$ not determined.

a Volcanic glass.
For all the sites, the Miocene/Pliocene boundary is marked by a shift in the elements that compose the noncarbonate phases, such as silicates and oxides. These noncarbonate fractions in the Miocene deposits contain higher $\mathrm{Fe}, \mathrm{Mn}$, and trace element contents than those from the overlying Pliocene deposits. For Site $\mathbf{5 2 0}$ only, iron and manganese are depleted in the siliceous layers, where silica, with calcite, is the second most prevalent element. The diatomite layers are enriched in $\mathrm{Pb}$ and $\mathrm{Zn}$. The iron crust at Site $\mathbf{5 2 0}$ has a very high content of $\mathrm{Si}$, and it has retained, with $\mathrm{Fe}$, trace elements such as $\mathrm{V}, \mathrm{Ni}, \mathrm{Co}, \mathrm{Cu}$, and $\mathrm{Zn}$. The lower part of the crust, composed of volcanogenic clasts, contains $\mathrm{Cr}$.

The difference between the Miocene sequences and the overlying Pliocene oozes can be related to a change in the origin and of the components of the noncarbonate fraction, as well as to the diagenetic evolution of these components. In the condensed clay sections of the upper Miocene units, the enrichment of iron, manganese, and trace elements is always well marked and re- 
Table 4. Major element chemistry (wt.\%) of sedimentary deposits, Site 520.

\begin{tabular}{|c|c|c|c|c|c|c|c|c|c|c|c|c|c|}
\hline $\begin{array}{l}\text { Core-Section } \\
\text { (interval in cm) }\end{array}$ & $\mathrm{SiO}_{2}$ & $\mathrm{Al}_{2} \mathrm{O}_{3}$ & $\mathrm{MgO}$ & $\mathrm{CaO}$ & $\mathrm{Fe}_{2} \mathrm{O}_{3}$ & $\mathrm{Mn}_{3} \mathrm{O}_{4}$ & $\mathrm{TiO}_{2}$ & $\mathrm{BaO}$ & $\mathrm{SrO}$ & $\mathrm{Na}_{2} \mathrm{O}$ & $\mathrm{K}_{2} \mathrm{O}$ & $\operatorname{Ign}^{a}$ & Total \\
\hline $9-12,131-133$ & 8.5 & 2.4 & 0.77 & 46.4 & 2.3 & 0.400 & 0.14 & 0.01 & 0.16 & 0.07 & $<0.05$ & 38.60 & 99.81 \\
\hline $9-2,138-140$ & 23.4 & 6.8 & 2.01 & 28.1 & 9.2 & 1.43 & 0.36 & 0.04 & 0.13 & 1.12 & 0.12 & 26.98 & 99.66 \\
\hline $11-4,55-59$ & 14.8 & 4.8 & 1.24 & 37.1 & 5.8 & 0.912 & 0.28 & 0.02 & 0.14 & 0.13 & $<0.05$ & 33.16 & 98.44 \\
\hline $14, \mathrm{CC}$ & 41.7 & 9.8 & 2.75 & 13.6 & 7.3 & 0.170 & 0.50 & 0.02 & 0.07 & 2.74 & 2.06 & 16.50 & 97.26 \\
\hline $15-2,31-33 A^{b}$ & 36.9 & 6.5 & 1.69 & 1.2 & 39.0 & 0.637 & 1.04 & 0.02 & 0.04 & 3.04 & 1.62 & 8.11 & 99.75 \\
\hline $15-2,31-33 B^{c}$ & 39.5 & 6.6 & 1.75 & 0.8 & 33.1 & 0.555 & 0.97 & 0.02 & 0.03 & 2.80 & 1.68 & 9.09 & 96.67 \\
\hline $15-2,31-33 C^{d}$ & 45.1 & 15.2 & 3.01 & 2.7 & 18.1 & 0.090 & 2.25 & 0.01 & 0.04 & 2.80 & 2.05 & 6.66 & 97.91 \\
\hline $15-2,38-40$ & 34.8 & 7.1 & 2.45 & 20.1 & 9.9 & 0.299 & 0.34 & 0.02 & 0.09 & 2.04 & 1.46 & 20.19 & 98.76 \\
\hline $15-2,42-44$ & 24.7 & 5.1 & 1.55 & 32.7 & 3.7 & 0.441 & 0.27 & 0.03 & 0.15 & 0.79 & 0.07 & 30.45 & 99.93 \\
\hline $16-4,20-22$ & 10.5 & 2.7 & 0.89 & 45.4 & 3.0 & 0.563 & 0.16 & 0.01 & 0.14 & 0.11 & $<0.05$ & 37.34 & 100.76 \\
\hline $16-4,27-28$ & 35.5 & 9.7 & 2.73 & 15.5 & 10.8 & 2.29 & 0.52 & 0.06 & 0.10 & 2.20 & 1.54 & 17.52 & 98.53 \\
\hline $26-1,22-24$ & 40.1 & 7.2 & 2.99 & 16.4 & 9.6 & 0.922 & 0.36 & 0.02 & 0.09 & 2.27 & 1.90 & 17.51 & 99.41 \\
\hline $26-1,116-119$ & 35.5 & 5.2 & 1.72 & 25.7 & 4.4 & 0.256 & 0.27 & 0.02 & 0.12 & 1.74 & 0.60 & 24.54 & 100.18 \\
\hline $29-1,136-139$ & 46.1 & 14.8 & 3.14 & 6.4 & 8.9 & 2.15 & 0.72 & 0.04 & 0.04 & 2.53 & 3.28 & 10.02 & 98.14 \\
\hline $29-1,147-149$ & 35.0 & 11.2 & 2.75 & 18.2 & 9.6 & 1.76 & 0.58 & 0.04 & 0.09 & 1.73 & 2.21 & 17.56 & 100.65 \\
\hline $29-2,13-16$ & 29.5 & 9.8 & 2.19 & 21.9 & 8.4 & 1.37 & 0.49 & 0.03 & 0.11 & 1.43 & 1.34 & 21.82 & 98.38 \\
\hline Diatomites & 56.7 & 1.5 & 0.58 & 14.7 & 3.8 & 0.103 & 0.05 & 0.02 & 0.07 & 2.04 & 0.50 & 18.56 & 98.60 \\
\hline
\end{tabular}

Note: Total iron is calculated as $\mathrm{Fe}_{2} \mathrm{O}_{3}$ and total manganese as $\mathrm{Mn}_{3} \mathrm{O}_{4}$.

a Ign = ignition at $1000^{\circ} \mathrm{C}$.

b Top crust.

c Mid-crust.

Table 5. Major element chemistry (wt.\%) of sedimentary deposits, Site 520, recalculated on CaO-free basis.

\begin{tabular}{|c|c|c|c|c|c|c|c|c|c|c|c|c|c|}
\hline $\begin{array}{l}\text { Core-Section } \\
\text { (interval in } \mathrm{cm} \text { ) }\end{array}$ & $\mathrm{SiO}_{2}$ & $\mathrm{Al}_{2} \mathrm{O}_{3}$ & $\mathrm{MgO}$ & $\mathrm{Fe}_{2} \mathrm{O}_{3}$ & $\mathrm{Mn}_{3} \mathrm{O}_{4}$ & $\mathrm{TiO}_{2}$ & $\mathrm{BaO}$ & $\mathrm{Na}_{2} \mathrm{O}$ & $\mathrm{K}_{2} \mathrm{O}$ & $\operatorname{Ign}^{a}$ & $\frac{\mathrm{SiO}_{2}}{\mathrm{Al}_{2} \mathrm{O}_{3}}$ & $\frac{\mathrm{Fe}_{2} \mathrm{O}_{4}}{\mathrm{Mn}_{3} \mathrm{O}_{4}}$ & $\mathrm{CaCO}_{3}{ }^{\mathrm{b}}$ \\
\hline $9-2,131-133$ & 50.65 & 14.30 & 4.59 & 13.70 & 2.38 & 0.83 & 0.06 & 0.42 & 0.30 & 12.77 & 3.54 & 5.75 & 82.8 \\
\hline $9-3,138-140$ & 49.39 & 13.77 & 4.07 & 18.63 & 2.90 & 0.73 & 0.08 & 2.27 & 0.24 & 9.93 & 3.58 & 6.42 & 50.2 \\
\hline $11-4,55-59$ & 46.16 & 14.97 & 3.93 & 18.09 & 2.84 & 0.87 & 0.06 & 0.41 & 0.16 & 12.51 & 3.08 & 6.37 & 66.2 \\
\hline $14, \mathrm{CC}$ & 57.24 & 13.45 & 3.77 & 10.02 & 0.23 & 0.69 & 0.03 & 3.76 & 2.83 & 7.98 & 4.26 & 43.57 & 24.2 \\
\hline $15-2,31-33 A^{c}$ & 37.80 & 6.66 & 1.73 & 39.95 & 0.65 & 1.07 & 0.02 & 3.11 & 1.66 & 7.34 & 5.68 & 61.46 & 2.1 \\
\hline $15-2,31-33 B^{d}$ & 41.39 & 6.92 & 1.83 & 34.68 & 0.58 & 1.02 & 0.02 & 2.93 & 1.76 & 8.87 & 5.98 & 59.79 & 1.4 \\
\hline $15-2,31-33 C^{\mathrm{e}}$ & 48.42 & 16.32 & 3.23 & 19.43 & 0.10 & 2.42 & 0.01 & 3.01 & 2.20 & 4.87 & 2.97 & 194.30 & 4.8 \\
\hline $15-2,38-40$ & 55.41 & 11.30 & 3.90 & 15.76 & 0.48 & 0.54 & 0.03 & 3.25 & 2.32 & 7.00 & 4.90 & 32.83 & 35.8 \\
\hline $15-2,42-44$ & 59.65 & 12.32 & 3.74 & 8.94 & 1.07 & 0.65 & 0.07 & 1.91 & 0.17 & 11.49 & 4.84 & 8.35 & 58.3 \\
\hline $16-4,20-22$ & 53.70 & 13.81 & 4.55 & 15.34 & 2.88 & 0.82 & 0.05 & 0.06 & 0.26 & 8.53 & 3.89 & 5.33 & 81.0 \\
\hline $16-4,27-28$ & 50.23 & 13.72 & 3.86 & 15.28 & 3.24 & 0.74 & 0.08 & 3.11 & 2.18 & 7.56 & 3.66 & 4.72 & 26.9 \\
\hline $26-1,22-24$ & 57.30 & 10.29 & 4.27 & 13.72 & 1.32 & 0.51 & 0.03 & 3.24 & 2.71 & 6.61 & 5.57 & 10.39 & 29.3 \\
\hline $26-1,116-119$ & 65.68 & 9.62 & 3.18 & 8.14 & 0.47 & 0.50 & 0.04 & 3.22 & 1.11 & 8.04 & 6.83 & 17.32 & 45.9 \\
\hline $29-1,136-139$ & 53.20 & 17.08 & 3.62 & 10.27 & 2.48 & 0.83 & 0.05 & 2.92 & 3.79 & 5.76 & 3.11 & 4.11 & 11.4 \\
\hline $29-1,147-149$ & 51.37 & 16.44 & 4.04 & 14.09 & 2.58 & 0.85 & 0.06 & 2.54 & 3.24 & 4.78 & 3.12 & 5.46 & 32.5 \\
\hline $29-2,13-16$ & 49.86 & 16.56 & 3.70 & 14.20 & 2.32 & 0.83 & 0.05 & 2.42 & 2.26 & 7.80 & 3.01 & 6.12 & 39.1 \\
\hline Diatomites & 78.42 & 2.07 & 0.80 & 5.26 & 0.14 & 0.07 & 0.03 & 2.82 & 0.69 & 9.70 & 37.88 & 37.57 & 26.2 \\
\hline
\end{tabular}

a $\operatorname{lgn}=$ ignition at $1000^{\circ} \mathrm{C}$

b Calculated from total $\mathrm{CaO}(\%)$.

c Top crust.

d Mid-crust.

Table 6. Trace element chemistry (ppm) of sedimentary deposits, Site 520.

\begin{tabular}{lrrrrrrrrrrr}
\hline $\begin{array}{c}\text { Core-Section } \\
\text { (interval in cm) }\end{array}$ & $\mathrm{Sr}$ & $\mathrm{Ba}$ & $\mathrm{V}$ & $\mathrm{Ni}$ & $\mathrm{Co}$ & $\mathrm{Cr}$ & $\mathrm{B}$ & $\mathrm{Zn}$ & $\mathrm{Ga}$ & $\mathrm{Cu}$ & $\mathrm{Pb}$ \\
\hline $9-2,131-133$ & 2011 & 106 & 43 & 37 & 23 & $<5$ & 12 & $<2$ & $<2$ & 49 & 20 \\
$9-3,138-140$ & 1335 & 592 & 197 & 124 & 77 & 36 & 110 & 73 & 11 & 131 & 121 \\
$11-4,55-59$ & 1441 & 215 & 79 & 114 & 64 & 21 & 58 & 22 & 2 & 92 & 21 \\
$14, \mathrm{CC}$ & 707 & 207 & 194 & 163 & 104 & 62 & - & 190 & - & 200 & - \\
$15-2,31-33 A^{\mathrm{a}}$ & 346 & 207 & 295 & 418 & 163 & 56 & - & 326 & - & 195 & - \\
$15-2,31-33 B^{\mathrm{b}}$ & 343 & 205 & 253 & 426 & 156 & 47 & - & 276 & - & 217 & - \\
$15-2,31-33 C^{\mathrm{C}}$ & 346 & 86 & 69 & 163 & 48 & 384 & - & 261 & - & 94 & - \\
$15-2,38-40$ & 796 & 150 & 244 & 152 & 65 & 45 & - & 127 & - & 82 & - \\
$15-2,42-44$ & 1889 & 284 & 183 & 80 & 40 & 25 & 68 & 39 & 5 & 88 & 111 \\
$16-4,20-22$ & 1581 & 149 & 61 & 59 & 27 & 12 & 41 & $<2$ & $<2$ & 39 & 28 \\
$16-4,27-28$ & 1695 & 762 & 221 & 189 & 213 & 63 & 150 & 129 & 12 & 164 & 214 \\
$26-1,22-24$ & 551 & 158 & 81 & 68 & 40 & 30 & 147 & 62 & 6 & 92 & 212 \\
$26-1,116-119$ & 1258 & 203 & 171 & 40 & 28 & 36 & 124 & 185 & 7 & 131 & 222 \\
$29-1,136-139$ & 891 & 466 & 149 & 310 & 167 & 65 & 197 & 146 & 40 & 270 & 269 \\
$29-1,147-149$ & 1584 & 394 & 250 & 203 & 160 & 69 & 218 & 89 & 14 & 126 & 159 \\
$29-2,13-16$ & 1106 & 424 & 207 & 173 & 125 & 55 & 170 & 113 & 15 & 98 & 138 \\
Diatomites & 451 & 158 & 33 & 143 & 107 & 29 & - & 65 & - & 33 & - \\
\hline
\end{tabular}

Note: Dashes $=$ not determined

Top crust.

c Mid-crust. flects the specific dissolution and early diagenesis processes.

Near the basement, the pelagic deposits contain increasing amounts of $\mathrm{Fe}, \mathrm{Mn}, \mathrm{Ti}, \mathrm{Ni}$, and $\mathrm{Cr}$. The presence of residual fresh basaltic products is indicated by $\mathrm{V}$ contents and a higher $\mathrm{V} / \mathrm{Fe}$ ratio (the comparison was made with values from basalt (see Carman, this vol.). The episodic volcanogenic components were also characterized by titanium and high $\mathrm{Ti} / \mathrm{Al}$ ratios referenced to basalts (Carman, this vol.), volcanic glass (e.g., Sample 519-37,CC) and other marine clays (Karpoff et al., 1981).

The diagenetic evolution of the clayey pelagic oozes of South Atlantic is also shown by a correlation diagram of calcium versus strontium (Fig. 8). Three types of samples are apparent: the calcareous oozes have a normal $\mathrm{Sr} / \mathrm{Ca}$ ratio (Turekian, 1964; Karpoff, 1980; Karpoff et al., 1981); the low $\mathrm{Sr} / \mathrm{Ca}$ ratio is characteristic of samples with a basaltic contribution; and the diagenetic en- 
Table 7. Major element chemistry (wt.\%) of sedimentary deposits, Site 521.

\begin{tabular}{|c|c|c|c|c|c|c|c|c|c|c|c|c|c|}
\hline $\begin{array}{l}\text { Core-Section } \\
\text { (interval in cm) }\end{array}$ & $\mathrm{SiO}_{2}$ & $\mathrm{Al}_{2} \mathrm{O}_{3}$ & $\mathrm{MgO}$ & $\mathrm{CaO}$ & $\mathrm{Fe}_{2} \mathrm{O}_{3}$ & $\mathrm{Mn}_{3} \mathrm{O}_{4}$ & $\mathrm{TiO}_{2}$ & $\mathrm{BaO}$ & $\mathrm{SrO}$ & $\mathrm{Na}_{2} \mathrm{O}$ & $\mathrm{K}_{2} \mathrm{O}$ & $\operatorname{Ign}^{a}$ & Total \\
\hline $7-1,74-76$ & 1.7 & 0.5 & 0.41 & 53.7 & 0.2 & 0.062 & 0.05 & 0.01 & 0.15 & 0.14 & $<0.05$ & 43.40 & 100.35 \\
\hline $11-1,35-37$ & 1.6 & 0.5 & 0.37 & 53.1 & $<0.1$ & 0.077 & 0.05 & $<0.01$ & 0.14 & 0.14 & $<0.05$ & 43.23 & 99.18 \\
\hline $11-1,130-132$ & 23.1 & 7.6 & 1.84 & 30.3 & 5.5 & 1.05 & 0.37 & 0.02 & 0.12 & 1.06 & 0.11 & 29.62 & 100.73 \\
\hline $11-2,10-12$ & 21.6 & 7.5 & 1.90 & 30.9 & 6.2 & 1.15 & 0.37 & 0.02 & 0.12 & 0.67 & 0.11 & 29.83 & 100.29 \\
\hline $11, \mathrm{CC}(15-17)$ & 27.0 & 9.6 & 2.28 & 22.2 & 8.6 & 1.58 & 0.48 & 0.03 & 0.11 & 1.82 & 0.44 & 24.19 & 98.43 \\
\hline $13-2,87-89$ & 4.0 & 1.4 & 0.54 & 51.1 & 1.2 & 0.208 & 0.09 & $<0.01$ & 0.14 & 0.10 & $<0.05$ & 41.82 & 100.50 \\
\hline $14-1,25-27$ & 5.4 & 1.9 & 0.60 & 48.6 & 1.4 & 0.249 & 0.12 & $<0.01$ & 0.15 & 0.07 & $<0.05$ & 40.75 & 99.25 \\
\hline $14-2,93-96$ & 35.6 & 12.0 & 2.55 & 16.2 & 8.4 & 1.56 & 0.56 & 0.04 & 0.08 & 2.03 & 1.63 & 18.67 & 99.37 \\
\hline $14, \mathrm{CC}(22-24)$ & 10.5 & 3.5 & 0.92 & 44.1 & 2.7 & 0.442 & 0.18 & 0.01 & 0.16 & 0.06 & $<0.05$ & 37.79 & 100.34 \\
\hline $16-1,22-25$ & 43.6 & 14.5 & 3.06 & 8.8 & 9.7 & 1.70 & 0.69 & 0.04 & 0.06 & 3.51 & 3.00 & 12.30 & 101.02 \\
\hline $16-2,112-114$ & 21.1 & 7.0 & 1.54 & 31.8 & 4.5 & 0.746 & 0.36 & 0.03 & 0.15 & 0.76 & 0.12 & 30.63 & 98.72 \\
\hline $19, \mathrm{CC}(20-23)$ & 21.0 & 6.7 & 1.60 & 32.5 & 5.8 & 0.816 & 0.37 & 0.02 & 0.15 & 0.36 & 0.10 & 31.32 & 100.77 \\
\hline
\end{tabular}

Note: Total iron is calculated as $\mathrm{Fe}_{2} \mathrm{O}_{3}$ and manganese as $\mathrm{Mn}_{3} \mathrm{O}_{4}$.

${ }^{2}$ Ign = ignition at $1000^{\circ} \mathrm{C}$.

Table 8. Major element chemistry (wt.\%) of sedimentary deposits, Site 521, recalculated on CaO-free basis.

\begin{tabular}{lccccccccccccc}
\hline $\begin{array}{c}\text { Core-Section } \\
\text { (interval in cm) }\end{array}$ & $\mathrm{SiO}_{2}$ & $\mathrm{Al}_{2} \mathrm{O}_{3}$ & $\mathrm{MgO}$ & $\mathrm{Fe}_{2} \mathrm{O}_{3}$ & $\mathrm{Mn}_{3} \mathrm{O}_{4}$ & $\mathrm{TiO}_{2}$ & $\mathrm{BaO}$ & $\mathrm{Na}_{2} \mathrm{O}$ & $\mathrm{K}_{2} \mathrm{O}$ & $\mathrm{Ign}^{\mathrm{a}}$ & $\frac{\mathrm{SiO}_{2}}{\mathrm{Al}_{2} \mathrm{O}_{3}}$ & $\frac{\mathrm{Fe}_{2} \mathrm{O}_{3}}{\mathrm{Mn}_{3} \mathrm{O}_{4}}$ & $\mathrm{CaCO}_{3} \mathrm{~b}$ \\
\hline $\mathbf{7 - 1 , 7 4 - 7 6}$ & 39.27 & 11.55 & 9.47 & 4.62 & 1.43 & 1.15 & 0.23 & 3.23 & 1.15 & 27.88 & 3.40 & 3.23 & 95.91 \\
$11-1,35-37$ & 36.32 & 11.35 & 8.40 & 2.27 & 1.75 & 1.13 & 0.23 & 3.18 & 1.13 & 34.24 & 3.20 & 1.30 & 94.84 \\
$11-1,130-132$ & 49.61 & 16.32 & 4.17 & 11.81 & 2.26 & 0.79 & 0.04 & 2.28 & 0.24 & 12.48 & 3.04 & 5.23 & 54.12 \\
$11-2,10-12$ & 47.84 & 16.61 & 4.21 & 13.73 & 2.55 & 0.82 & 0.04 & 1.48 & 0.24 & 12.47 & 2.88 & 5.38 & 55.29 \\
$11, \mathrm{CC}(15-17)$ & 46.16 & 16.41 & 3.90 & 14.70 & 2.70 & 0.68 & 0.05 & 3.11 & 0.75 & 11.53 & 2.81 & 5.44 & 39.65 \\
$13-2,87-89$ & 43.16 & 15.11 & 5.83 & 12.95 & 2.24 & 0.97 & 0.11 & 1.08 & 0.54 & 18.02 & 2.86 & 5.78 & 91.26 \\
$14-1,25-27$ & 42.68 & 15.37 & 4.85 & 11.32 & 2.01 & 0.97 & 0.08 & 0.57 & 0.40 & 20.74 & 2.84 & 5.63 & 86.80 \\
$14-2,93-96$ & 50.63 & 17.07 & 3.63 & 11.95 & 2.22 & 0.80 & 0.06 & 2.89 & 2.32 & 8.45 & 2.97 & 5.38 & 28.93 \\
$14, C \mathrm{C}(22-24)$ & 48.83 & 16.28 & 4.28 & 12.56 & 2.06 & 0.84 & 0.05 & 0.28 & 0.23 & 14.60 & 3.00 & 4.83 & 78.76 \\
$16-1,22-25$ & 51.18 & 17.02 & 3.59 & 11.39 & 2.00 & 0.81 & 0.05 & 4.12 & 3.52 & 6.32 & 3.01 & 5.70 & 15.72 \\
$16-2,112-114$ & 50.48 & 16.75 & 3.68 & 10.77 & 1.78 & 0.86 & 0.07 & 1.82 & 0.29 & 13.50 & 3.01 & 6.05 & 56.72 \\
$19, C C(20-23)$ & 49.36 & 15.75 & 3.76 & 13.63 & 1.92 & 0.97 & 0.05 & 0.85 & 0.24 & 13.57 & 3.13 & 8.66 & 58.05 \\
\hline
\end{tabular}

a Ign = ignition at $1000^{\circ} \mathrm{C}$.

b Calculated from total $\mathrm{CaO}(\%)$.

Table 9. Trace element chemistry (ppm) of sedimentary deposits, Site 521.

\begin{tabular}{lrrrrrrrrrrr}
\hline $\begin{array}{c}\text { Core-Section } \\
\text { (interval in cm) }\end{array}$ & $\mathrm{Sr}$ & $\mathrm{Ba}$ & $\mathrm{V}$ & $\mathrm{Ni}$ & $\mathrm{Co}$ & $\mathrm{Cr}$ & $\mathrm{B}$ & $\mathrm{Zn}$ & $\mathrm{Ga}$ & $\mathrm{Cu}$ & $\mathrm{Pb}$ \\
\hline $7-1,74-76$ & 2331 & 146 & $<5$ & 14 & 2 & 8 & 26 & $<2$ & $<2$ & 9 & 13 \\
$11-1,35-37$ & 1578 & 23 & $<5$ & 2 & $<2$ & $<5$ & $<5$ & $<2$ & $<2$ & 7 & 10 \\
$11-1,130-132$ & 1348 & 299 & 101 & 177 & 120 & 38 & 117 & 76 & 10 & 73 & 81 \\
$11-2,10-12$ & 1498 & 341 & 131 & 156 & 121 & 39 & 124 & 80 & 12 & 86 & 163 \\
$11, \mathrm{CC}(15-17)$ & 1412 & 393 & 174 & 225 & 160 & 43 & 139 & 104 & 14 & 49 & 120 \\
$13-2,87-89$ & 2059 & 75 & 41 & 32 & 19 & $<5$ & 36 & $<2$ & $<2$ & 9 & 26 \\
$14-1,25-27$ & 1858 & 74 & 26 & 35 & 19 & 5 & 18 & $<2$ & $<2$ & 7 & 26 \\
$14-2,93-96$ & 816 & 543 & 167 & 198 & 151 & 61 & 148 & 148 & 14 & 48 & 218 \\
$14, \mathrm{CC}(22-24)$ & 1964 & 124 & 39 & 63 & 36 & 10 & 21 & 9 & $<2$ & 46 & 33 \\
$16-1,22-25$ & 712 & 692 & 264 & 246 & 174 & 87 & 234 & 127 & 25 & 111 & 262 \\
$16-2,112-114$ & 1339 & 232 & 64 & 120 & 69 & 31 & 87 & 62 & 9 & 58 & 241 \\
$19, \mathrm{CC}(20-23)$ & 1952 & 249 & 111 & 107 & 34 & 36 & 106 & 75 & 10 & 69 & 161 \\
& & & & & & & & & & &
\end{tabular}

richment in $\mathrm{Sr}$ is well marked in the samples that contain the most clay minerals and have many micronodules in their coarse fraction. The strontium released from carbonates by dissolution is retained in the authigenic oxides and less in the transformed clay minerals.

\section{Clay Fractions}

Geochemical analyses were made on some clay fractions (Tables 16 and 17). They generally reflect the mixture of clay minerals with prevalent smectites. A good correlation exists between the fine fraction composition and the calculated noncarbonate phase composition. ${ }^{2}$ The more illitic samples give higher $\mathrm{K}$ contents. The fine fractions from basal sediments are generally enriched in

\footnotetext{
${ }^{2}$ Except for $\mathrm{Na}_{2} \mathrm{O}$ contents, which are explained by pollution by seawater salts in the
} bulk sediments; the values are lower in the clay fraction washed for extraction. magnesium. All the fine fractions are iron and manganese rich, and they retain high contents of $\mathrm{V}, \mathrm{Zn}, \mathrm{Cu}$, and $\mathrm{Ni}$.

The clay fraction from Sample $520-15-2,38-40 \mathrm{~cm}$ below the iron crust comprises $90 \%$ smectites. The structural formula of these smectites can be obtained as follows:

$$
\begin{gathered}
\left(\mathrm{Si}_{3.84}, \mathrm{Al}_{0.16}\right)\left(\mathrm{Al}_{0.76}, \mathrm{Fe}_{0.88}, \mathrm{Mg}_{0.33}, \mathrm{Ti}_{0.03}\right) \mathrm{O}_{10}(\mathrm{OH})_{2} \\
\text { Interlayered cations: }\left(\mathrm{Ca}_{0.1}, \mathrm{Na}_{0.05}, \mathrm{~K}_{0.2}\right)
\end{gathered}
$$

This formula is characteristic of a dioctahedral smectite such as nontronite and is similar to that of authigenic clay formed under volcanic influence (Karpoff et al., 1981).

The fine fractions from Samples 522A-30-2, 43-45 $\mathrm{cm}$ and 522B-3-2, 100-105 cm from the Eocene basal marly calcareous oozes are also composed of prevalent smectites. They have the structural formulae of ironbeideillites and contain more $\mathrm{Mg}$ and $\mathrm{Al}$ than the previous nontronite:

\section{$\left(\mathrm{Si}_{3.59}, \mathrm{Al}_{0.4}\right)\left(\mathrm{Al}_{0.55}, \mathrm{Fe}_{0.82}, \mathrm{Mg}_{0.66}, \mathrm{Ti}_{0.04}\right) \mathrm{O}_{10}(\mathrm{OH})_{2}$ Interlayered cations: $\left(\mathrm{Ca}_{0.11}, \mathrm{Na}_{0.05}, \mathrm{~K}_{0.25}\right)$}

The sample nearest to basalt (522B-3-2, 100-105 cm) contains also in its fine fraction high contents of $\mathrm{Mn}$, $\mathrm{Ni}, \mathrm{K}$, and $\mathrm{Ba}$, resulting from volcanogenic compounds (oxides, small silicate clasts).

The clays from the sediments overlying the basalt are $\mathrm{Mg}$ and $\mathrm{Ti}$ rich; their $\mathrm{Zn}, \mathrm{Cu}$, and $\mathrm{V}$ contents appear 
Table 10. Major element chemistry (wt.\%) of sedimentary deposits, Site 522.

\begin{tabular}{|c|c|c|c|c|c|c|c|c|c|c|c|c|c|}
\hline $\begin{array}{l}\text { Core-Section } \\
\text { (interval in } \mathrm{cm} \text { ) }\end{array}$ & $\mathrm{SiO}_{2}$ & $\mathrm{Al}_{2} \mathrm{O}_{3}$ & $\mathrm{MgO}$ & $\mathrm{CaO}$ & $\mathrm{Fe}_{2} \mathrm{O}_{3}$ & $\mathrm{Mn}_{3} \mathrm{O}_{4}$ & $\mathrm{TiO}_{2}$ & $\mathrm{BaO}$ & $\mathrm{SrO}$ & $\mathrm{Na}_{2} \mathrm{O}$ & $\mathrm{K}_{2} \mathrm{O}$ & $\operatorname{Ign}^{a}$ & Total \\
\hline $1-1,54-56$ & 11.3 & 3.7 & 1.03 & 42.5 & 2.2 & 0.345 & 0.18 & 0.05 & 0.14 & 0.06 & $<0.05$ & 38.11 & 99.54 \\
\hline $8-2,132-134$ & 3.7 & 1.2 & 0.61 & 50.6 & 0.5 & 0.128 & 0.08 & 0.01 & 0.13 & 0.07 & $<0.05$ & 42.31 & 99.36 \\
\hline $9-2,18-22$ & 14.3 & 4.5 & 1.12 & 40.9 & 2.6 & 0.503 & 0.23 & 0.01 & 0.15 & 0.05 & $<0.05$ & 35.96 & 100.37 \\
\hline $9-2,134-140$ & 47.7 & 15.4 & 3.49 & 6.0 & 9.4 & 1.66 & 0.69 & 0.05 & 0.05 & 3.07 & 3.29 & 10.17 & 100.99 \\
\hline $9, \mathrm{CC}$ & 42.1 & 12.3 & 2.90 & 9.8 & 8.4 & 1.43 & 0.56 & 0.04 & 0.06 & 2.63 & 2.81 & 13.48 & 96.44 \\
\hline $10-2,15-17$ & 52.1 & 16.6 & 3.57 & 1.3 & 9.9 & 1.51 & 0.75 & 0.05 & 0.03 & 3.44 & 3.83 & 6.73 & 99.78 \\
\hline $10-3,14-18$ & 50.7 & 14.7 & 3.21 & 1.8 & 9.3 & 1.33 & 0.68 & 0.03 & 0.02 & 3.07 & 3.76 & 7.27 & 95.77 \\
\hline $11-1,74-76$ & 52.6 & 17.1 & 3.43 & 1.2 & 9.6 & 1.43 & 0.79 & 0.05 & 0.03 & 2.91 & 3.77 & 6.96 & 99.81 \\
\hline $11-2,114-116$ & 53.3 & 17.6 & 3.44 & 1.3 & 9.1 & 1.36 & 0.78 & 0.05 & 0.02 & 2.88 & 3.91 & 6.69 & 100.34 \\
\hline $11, \mathrm{CC}(10-12)$ & 53.1 & 15.4 & 3.30 & 1.3 & 9.2 & 1.33 & 0.70 & 0.04 & 0.03 & 2.90 & 3.91 & 6.97 & 98.22 \\
\hline $12-2,14-16$ & 10.8 & 3.4 & 0.91 & 44.6 & 1.7 & 0.258 & 0.18 & 0.01 & 0.18 & 0.51 & 0.06 & 38.01 & 100.66 \\
\hline $12-3,4-6$ & 35.8 & 11.6 & 2.46 & 18.7 & 5.7 & 0.645 & 0.54 & 0.03 & 0.11 & 1.75 & 2.01 & 19.99 & 99.44 \\
\hline $14-1,94-96$ & 41.2 & 13.5 & 2.86 & 12.4 & 8.2 & 1.40 & 0.60 & 0.04 & 0.09 & 2.45 & 2.53 & 15.60 & 100.89 \\
\hline $15-2,50-52$ & 9.9 & 3.2 & 0.81 & 44.8 & 1.6 & 0.188 & 0.17 & 0.02 & 0.16 & 0.39 & $<0.05$ & 38.41 & 99.68 \\
\hline $25-3,25-27$ & 6.2 & 2.0 & 0.62 & 48.9 & 1.0 & 0.110 & 0.12 & 0.03 & 0.17 & 0.24 & $<0.05$ & 40.61 & 99.95 \\
\hline $30-3,90-92$ & 4.1 & 1.2 & 0.55 & 50.0 & 0.7 & 0.117 & 0.09 & 0.02 & 0.15 & 0.16 & $<0.05$ & 41.84 & 99.05 \\
\hline $34, \mathrm{CC}(7-10)$ & 3.3 & 1.0 & 0.50 & 51.1 & 0.9 & 0.216 & 0.08 & 0.02 & 0.13 & 0.13 & $<0.05$ & 42.10 & 99.59 \\
\hline $36, \mathrm{CC}(15-20)$ & 5.3 & 1.5 & 0.62 & 49.9 & 1.2 & 0.255 & 0.10 & 0.02 & 0.14 & 0.19 & $<0.05$ & 40.97 & 100.21 \\
\hline $37, \mathrm{CC}(7-11)$ & 3.6 & 1.0 & 0.54 & 51.0 & 1.8 & 0.250 & 0.08 & 0.02 & 0.13 & 0.13 & $<0.05$ & 41.95 & 100.57 \\
\hline $30 \mathrm{~A}-2,43-45$ & 18.9 & 5.0 & 3.23 & 33.8 & 5.2 & 0.380 & 0.40 & 0.01 & 0.10 & 1.19 & 0.43 & 30.22 & 98.96 \\
\hline $31 \mathrm{~A}-1,85-87$ & 6.4 & 1.4 & 0.76 & 44.9 & 3.1 & 0.938 & 0.09 & 0.01 & 0.14 & 0.05 & $<0.05$ & 39.51 & 97.41 \\
\hline $31 \mathrm{~A}, \mathrm{CC}(5-7)$ & 5.8 & 1.5 & 0.81 & 47.3 & 2.3 & 0.788 & 0.11 & 0.02 & 0.15 & 0.22 & $<0.05$ & 40.19 & 99.42 \\
\hline $3 \mathrm{~B}-1,120-122$ & 4.3 & 1.2 & 0.61 & 49.8 & 1.9 & 0.549 & 0.09 & 0.02 & 0.13 & 0.15 & $<0.05$ & 41.11 & 99.79 \\
\hline $3 B-2,100-102$ & 7.0 & 1.8 & 0.83 & 47.8 & 2.3 & 0.605 & 0.13 & 0.02 & 0.13 & 0.35 & $<0.05$ & 39.14 & 100.27 \\
\hline
\end{tabular}

Note: Total iron is calculated as $\mathrm{Fe}_{2} \mathrm{O}_{3}$ and total manganese as $\mathrm{Mn}_{3} \mathrm{O}_{4}$.

${ }^{a}$ Ign = ignition at $1000^{\circ} \mathrm{C}$.

Table 11. Major element chemistry (wt.\%) of sedimentary deposits, Site 522, recalculated on CaO-free basis.

\begin{tabular}{|c|c|c|c|c|c|c|c|c|c|c|c|c|c|}
\hline $\begin{array}{c}\text { Sample } \\
\text { (interval in } \mathrm{cm} \text { ) }\end{array}$ & $\mathrm{SiO}_{2}$ & $\mathrm{Al}_{2} \mathrm{O}_{3}$ & $\mathrm{MgO}$ & $\mathrm{Fe}_{2} \mathrm{O}_{3}$ & $\mathrm{Mn}_{3} \mathrm{O}_{4}$ & $\mathrm{TiO}_{2}$ & $\mathrm{BaO}$ & $\mathrm{Na}_{2} \mathrm{O}$ & $\mathrm{K}_{2} \mathrm{O}$ & $\operatorname{Ign}^{\mathrm{a}}$ & $\frac{\mathrm{SiO}_{2}}{\mathrm{Al}_{2} \mathrm{O}_{3}}$ & $\frac{\mathrm{Fe}_{2} \mathrm{O}_{3}}{\mathrm{Mn}_{3} \mathrm{O}_{4}}$ & $\mathrm{CaCO}_{3}{ }^{\mathrm{b}}$ \\
\hline $1-1,54-56$ & 47.82 & 15.66 & 4.36 & 9.31 & 1.46 & 0.76 & 0.21 & 0.25 & 0.21 & 19.96 & 3.05 & 6.38 & 75.9 \\
\hline $8-2,132-134$ & 41.47 & 13.48 & 6.85 & 5.62 & 1.44 & 0.90 & 0.11 & 0.79 & 0.56 & 28.68 & 3.08 & 3.90 & 90.3 \\
\hline $9-2,18-22$ & 52.60 & 16.55 & 4.12 & 9.56 & 1.85 & 0.85 & 0.04 & 0.18 & 0.18 & 14.07 & 3.18 & 5.17 & 73.0 \\
\hline $9-2,134-140$ & 52.88 & 17.07 & 3.87 & 10.42 & 1.84 & 0.76 & 0.06 & 3.40 & 3.65 & 6.07 & 3.10 & 5.37 & 10.7 \\
\hline $9, \mathrm{CC}$ & 54.32 & 15.58 & 3.67 & 10.64 & 1.81 & 0.71 & 0.05 & 3.33 & 3.56 & 7.32 & 3.42 & 5.87 & 17.5 \\
\hline $10-2,15-17$ & 53.46 & 17.03 & 3.66 & 10.16 & 1.55 & 0.77 & 0.05 & 3.53 & 3.93 & 5.86 & 3.14 & 6.55 & 2.3 \\
\hline $10-3,14-18$ & 54.73 & 15.87 & 3.47 & 10.04 & 1.44 & 0.73 & 0.03 & 3.31 & 4.06 & 6.32 & 3.45 & 6.99 & 3.2 \\
\hline $11-1,74-76$ & 53.84 & 17.50 & 3.51 & 9.83 & 1.46 & 0.81 & 0.05 & 2.98 & 3.86 & 6.16 & 3.05 & 6.73 & 2.1 \\
\hline $11-2,114-116$ & 54.34 & 17.94 & 3.51 & 9.28 & 1.39 & 0.80 & 0.05 & 2.94 & 3.99 & 5.78 & 3.03 & 6.68 & 2.3 \\
\hline $11, \mathrm{CC}(10-12)$ & 55.41 & 16.07 & 3.44 & 9.60 & 1.39 & 0.73 & 0.04 & 3.03 & 4.08 & 6.21 & 3.44 & 6.91 & 2.3 \\
\hline $12-2,14-16$ & 51.94 & 16.35 & 4.38 & 8.17 & 1.24 & 0.87 & 0.05 & 2.45 & 0.29 & 14.27 & 3.18 & 6.59 & 79.6 \\
\hline $12-3,4-6$ & 54.38 & 17.62 & 3.74 & 8.66 & 0.98 & 0.82 & 0.05 & 2.66 & 3.05 & 8.05 & 3.09 & 8.84 & 33.4 \\
\hline $14-1,94-96$ & 52.39 & 17.17 & 3.64 & 10.43 & 1.78 & 0.76 & 0.05 & 3.12 & 3.22 & 7.45 & 3.05 & 5.86 & 22.1 \\
\hline $15-2,50-52$ & 50.67 & 16.38 & 4.15 & 8.19 & 0.96 & 0.87 & 0.10 & 2.00 & 0.26 & 16.43 & 3.09 & 8.53 & 80.0 \\
\hline $25-3,25-27$ & 49.37 & 15.93 & 4.94 & 7.96 & 0.88 & 0.96 & 0.24 & 1.91 & 0.40 & 17.43 & 3.23 & 9.05 & 87.3 \\
\hline $30-3,90-92$ & 42.97 & 12.58 & 5.76 & 7.34 & 1.23 & 0.94 & 0.21 & 1.68 & 0.52 & 26.77 & 3.42 & 5.97 & 89.3 \\
\hline $34, \mathrm{CC}(7-10)$ & 40.51 & 12.28 & 6.14 & 11.05 & 2.65 & 0.98 & 0.25 & 1.60 & 0.61 & 23.94 & 3.30 & 4.17 & 91.2 \\
\hline $36, \mathrm{CC}(15-20)$ & 48.19 & 13.64 & 5.64 & 10.91 & 2.32 & 0.91 & 0.18 & 1.73 & 0.45 & 16.03 & 3.53 & 4.70 & 82.1 \\
\hline $37, \mathrm{CC}(7-11)$ & 53.45 & 8.10 & 4.37 & 14.58 & 2.02 & 0.62 & 0.16 & 1.05 & 0.40 & 15.21 & 6.60 & 7.22 & 91.0 \\
\hline $30 \mathrm{~A}-2,43-45$ & 49.22 & 13.02 & 8.41 & 13.54 & 0.99 & 1.04 & 0.03 & 3.10 & 1.12 & 9.54 & 3.78 & 13.68 & 60.3 \\
\hline $31 \mathrm{~A}-1,85-87$ & 37.58 & 8.22 & 4.46 & 18.20 & 5.51 & 0.53 & 0.06 & 0.29 & 0.29 & 24.85 & 4.57 & 3.30 & 80.2 \\
\hline $31 \mathrm{~A}, \mathrm{CC}(5-7)$ & 39.13 & 10.12 & 5.46 & 16.86 & 5.32 & 0.74 & 0.13 & 1.48 & 0.34 & 20.41 & 3.77 & 3.17 & 84.4 \\
\hline $3 \mathrm{~B}-1,120-122$ & 39.63 & 11.06 & 5.62 & 17.51 & 5.06 & 0.83 & 0.18 & 1.38 & 0.46 & 18.26 & 3.58 & 3.46 & 88.9 \\
\hline $3 B-2,100-102$ & 47.72 & 12.27 & 5.66 & 15.68 & 4.12 & 0.89 & 0.14 & 2.39 & 0.34 & 10.79 & 3.89 & 3.81 & 85.3 \\
\hline
\end{tabular}

${ }^{\mathrm{a}}$ Ign $=$ ignition at $1000^{\circ} \mathrm{C}$.

b Calculated from total $\mathrm{CaO}(\%)$.

slightly higher than those of the red clay layers (Tables 16 and 17, first set of samples). These facts have been described by Melson and Thompson (1973). The different mineral types and complex clays formed from basalt and volcanic glass alteration at low temperatures are described by Hajash (1975) and Honnorez (1981).

\section{Discussion}

The geochemical investigation of the pelagic clay from the eastern flank of the Mid-Atlantic Ridge at $-26^{\circ} \mathrm{S}$ reveals that both oceanographic factors, which controlled the dissolution of the biogenic calcareous oozes, and basalt alteration affected the chemical patterns of the resulting red clay facies. The relative concentrations of the major elements, particularly iron and manganese, result from both influences; higher values of $\mathrm{Mg}, \mathrm{Ti}, \mathrm{V}$, and $\mathrm{Cr}$ in authigenic clays and oxides characterize the basaltic/volcanic contribution. The specific contribution of the biogenic phases to authigenesis is marked by $\mathrm{Sr}$ and $\mathrm{Zn}$. Boyle (1981) determined that calcareous biogenic phases are carriers for trace elements, particularly $\mathrm{Zn}$. The biogenic origin of $\mathrm{Ba}$ and $\mathrm{Cu}$ in marine authigenic phases has often been proposed (El Wakeel and Riley, 1961; Chow and Goldberg, 1970; Karpoff et al., 1981). For the other trace elements, such as $\mathrm{Ni}, \mathrm{Co}$, and $\mathrm{Pb}$, it is difficult to separate these strongly associated factors in these sedimentary sequences. Indeed, the dissolution processes result in the concentration of the detrital minerals as much as the sporadic volcaniclastic compounds; these later can promote, in their turn, the transformation and authigeneses of clays and oxides. 
Table 12. Trace element chemistry (ppm) of sedimentary deposits, Site 522.

\begin{tabular}{lrrrrrrrrrr}
\hline $\begin{array}{c}\text { Core-Section } \\
\text { (interval in cm) }\end{array}$ & $\mathrm{Sr}$ & $\mathrm{Ba}$ & $\mathrm{V}$ & $\mathrm{Ni}$ & $\mathrm{Co}$ & $\mathrm{Cr}$ & $\mathrm{B}$ & $\mathrm{Zn}$ & $\mathrm{Cu}$ & $\mathrm{Pb}$ \\
\hline $1-1,54-56$ & 1781 & 587 & 31 & 66 & 27 & 21 & 59 & 4 & 55 & 32 \\
$8-2,132-134$ & 1539 & 131 & 8 & 25 & 12 & $<5$ & $<5$ & $<2$ & 11 & 19 \\
$9-2,18-22$ & 1740 & 133 & 29 & 109 & 53 & 22 & 65 & 8 & 65 & 39 \\
$9-2,134-140$ & 555 & 507 & 98 & 359 & 149 & 60 & 164 & 121 & 219 & 227 \\
$9, \mathrm{CC}$ & 520 & 362 & 125 & 224 & 138 & 67 & - & 138 & 158 & - \\
$10-2,15-17$ & 354 & 435 & 127 & 315 & 142 & 87 & 217 & 132 & 206 & 313 \\
$10-3,14-18$ & 247 & 384 & 141 & 206 & 135 & 69 & - & 163 & 139 & - \\
$11-1,74-76$ & 322 & 392 & 134 & 275 & 131 & 79 & 218 & 124 & 181 & 131 \\
$11-2,114-116$ & 320 & 421 & 132 & 284 & 123 & 70 & 229 & 132 & 168 & 149 \\
$11, \mathrm{CC}(10-12)$ & 251 & 421 & 147 & 199 & 120 & 63 & - & 163 & 130 & - \\
$12-2,14-16$ & 2298 & 90 & 31 & 45 & 20 & 5 & 40 & $<2$ & 31 & 16 \\
$12-3,4-6$ & 1132 & 396 & 123 & 163 & 74 & 63 & 166 & 92 & 46 & 43 \\
$14-1,94-96$ & 1241 & 468 & 167 & 182 & 119 & 48 & 190 & 115 & 53 & 64 \\
$15-2,50-52$ & 1738 & 212 & 11 & 29 & 3 & $<5$ & 14 & 2 & 46 & 6 \\
$25-3,25-27$ & 1934 & 357 & 30 & 9 & $<2$ & $<5$ & 8 & $<2$ & 22 & 10 \\
$30-3,90-92$ & 1897 & 240 & $<5$ & 6 & $<2$ & $<5$ & $<5$ & $<2$ & 14 & $<2$ \\
$34, C C(7-10)$ & 1490 & 253 & 33 & 21 & $<2$ & $<5$ & 7 & $<2$ & 20 & 17 \\
$36, C C(15-20)$ & 1453 & 188 & 38 & 20 & $<2$ & $<5$ & 9 & $<2$ & 27 & 21 \\
$37, C C(7-11)$ & 1701 & 139 & 15 & 18 & $<2$ & $<5$ & $<5$ & $<2$ & 15 & 12 \\
$30 A-2,43-45$ & 835 & 111 & 43 & 53 & 19 & 23 & 27 & 132 & 77 & 34 \\
$31 A-1,85-87$ & 1301 & 121 & 64 & 119 & 34 & 17 & - & 64 & 50 & - \\
$31 A-C C ~(5-7)$ & 1640 & 169 & 42 & 82 & $<2$ & $<5$ & $<5$ & 13 & 88 & 36 \\
$3 B-1,120-122$ & 1714 & 241 & 43 & 50 & 4 & $<5$ & 8 & 3 & 44 & 30 \\
$3 B-2,100-102$ & 1297 & 190 & 46 & 47 & 2 & $<5$ & 7 & 17 & 54 & 15 \\
\hline
\end{tabular}

Note: Dashes $=$ not determined.

The beginning of dissolution is marked by the early precipitation of manganese oxides as small black spots on or within the foraminifer tests; the manganese subsequently forms micronodules. Marine authigenesis of iron-manganese oxyhydroxides is well known and has been described at varying depths and environments (Arrhenius, 1963; Glasby, 1977 and 1978; Coll. Int. CNRS,
1979). The most common hypothesis for the formation of ferromanganese oxides is precipitation from seawater which is easier in oxidized, cool, deep water under volcanic/basaltic influences. In the condensed South Atlantic sedimentary sequences, particularly through the upper Miocene (Sites 519 to 523), the formation of manganese oxide can be related to cooling periods; in the basal sediments of Miocene and Eocene age (Sites $519,520,522$, and 523), it can also be related to basaltic weathering.

The sole example of iron crust at Site 520 (Plate 6) shows that in a siliceous environment, under light volcanic influence, the precipitation of iron is accompanied by the concentration of $\mathrm{Ni}, \mathrm{Co}, \mathrm{Zn}, \mathrm{Cu}$, and $\mathrm{V}$ without Mn. By its colloform structure and composition, the crust fragment at Site $\mathbf{5 2 0}$ gives a particularly good example of a type of concretion intermediate between manganiferous concretions, crust, and indurated levels on the one hand (Hoffert et al., 1978; Coll. Int. CNRS, 1979; Karpoff et al., 1980) and siliceous clay concretion (Karpoff et al., 1981) on the other. Both types of concretion form under volcanic influences at low accumulation rates or during hiatus periods.

\section{THE FORMATION OF RED CLAY FACIES IN THE SOUTH ATLANTIC: CLAY AND OXIDE GENESES}

\section{Clays}

In the pelagic deposits from Sites 519 to 523 , the noncarbonate phase includes clays that are detrital, transformed, and authigenic. The low clay fractions from the

Table 13. Major element chemistry (wt.\%) of sedimentary deposits, Site 523.

\begin{tabular}{|c|c|c|c|c|c|c|c|c|c|c|c|c|c|}
\hline $\begin{array}{l}\text { Core-Section } \\
\text { (interval in cm) }\end{array}$ & $\mathrm{SiO}_{2}$ & $\mathrm{Al}_{2} \mathrm{O}_{3}$ & $\mathrm{MgO}$ & $\mathrm{CaO}$ & $\mathrm{Fe}_{2} \mathrm{O}_{3}$ & $\mathrm{Mn}_{3} \mathrm{O}_{4}$ & $\mathrm{TiO}_{2}$ & $\mathrm{BaO}$ & $\mathrm{SrO}$ & $\mathrm{Na}_{2} \mathrm{O}$ & $\mathrm{K}_{2} \mathrm{O}$ & $\lg ^{a}$ & Total \\
\hline $8-3,62-64$ & 37.7 & 12.2 & 2.63 & 15.0 & 6.5 & 0.964 & 0.57 & 0.04 & 0.08 & 2.00 & 1.92 & 17.89 & 97.39 \\
\hline $10-1,134-139$ & 51.8 & 16.2 & 3.55 & 1.4 & 8.9 & 1.23 & 0.75 & 0.05 & 0.03 & 2.91 & 3.53 & 7.18 & 97.48 \\
\hline $11-2,54-58$ & 50.1 & 16.0 & 3.02 & 5.5 & 7.2 & 0.931 & 0.71 & 0.08 & 0.05 & 3.28 & 4.00 & 9.69 & 100.58 \\
\hline $20-2,115-117$ & 25.3 & 7.8 & 1.79 & 30.2 & 3.4 & 0.327 & 0.39 & 0.07 & 0.13 & 1.28 & 0.95 & 27.56 & 99.24 \\
\hline $22, \mathrm{CC}$ & 7.7 & 2.3 & 0.69 & 48.6 & 0.9 & 0.084 & 0.14 & 0.03 & 0.18 & 0.28 & 0.05 & 39.58 & 100.51 \\
\hline $28-2,62-64$ & 30.0 & 9.0 & 2.18 & 27.1 & 4.5 & 0.438 & 0.46 & 0.04 & 0.12 & 1.34 & 1.30 & 24.51 & 101.05 \\
\hline $36, \mathrm{CC}(25-26)$ & 14.5 & 4.1 & 1.21 & 40.9 & 2.7 & 0.356 & 0.25 & 0.02 & 0.14 & 0.75 & 0.07 & 35.05 & 99.98 \\
\hline $37-3,45-46$ & 40.7 & 11.8 & 3.08 & 10.8 & 9.3 & 1.94 & 0.65 & 0.14 & 0.07 & 2.40 & 2.92 & 14.08 & 97.95 \\
\hline $37, \mathrm{CC}(10-12)$ & 22.1 & 6.4 & 1.72 & 33.1 & 4.3 & 0.745 & 0.36 & 0.03 & 0.12 & 1.13 & 0.61 & 29.97 & 100.64 \\
\hline $44-3,3-5$ & 6.3 & 1.7 & 0.68 & 49.0 & 1.4 & 0.187 & 0.14 & 0.03 & 0.15 & 0.23 & $<0.05$ & 40.48 & 100.31 \\
\hline $50-2,145-148$ & 6.3 & 1.5 & 0.65 & 48.2 & 1.1 & 0.149 & 0.16 & 0.01 & 0.12 & 0.24 & $<0.05$ & 40.64 & 99.22 \\
\hline $50-3,25-28$ & 5.1 & 1.3 & 0.65 & 49.8 & 1.1 & 0.316 & 0.11 & 0.02 & 0.12 & 0.19 & $<0.05$ & 41.23 & 99.92 \\
\hline
\end{tabular}

Note: Total iron is calculated as $\mathrm{Fe}_{2} \mathrm{O}_{3}$ and total manganese as $\mathrm{Mn}_{3} \mathrm{O}_{4}$.

a $\mathrm{Ign}=$ ignition at $1000^{\circ} \mathrm{C}$.

Table 14. Major element chemistry (wt.\%) of sedimentary deposits, Site 523, recalculated on CaO-free basis.

\begin{tabular}{lccccccccccccr}
\hline $\begin{array}{c}\text { Core-Section } \\
\text { (interval in cm) }\end{array}$ & $\mathrm{SiO}_{3}$ & $\mathrm{Al}_{2} \mathrm{O}_{3}$ & $\mathrm{MgO}$ & $\mathrm{Fe}_{2} \mathrm{O}_{3}$ & $\mathrm{Mn}_{3} \mathrm{O}_{4}$ & $\mathrm{TiO}_{2}$ & $\mathrm{BaO}$ & $\mathrm{Na}_{2} \mathrm{O}$ & $\mathrm{K}_{2} \mathrm{O}$ & $\mathrm{Ign}^{\mathrm{a}}$ & $\frac{\mathrm{Si}_{2} \mathrm{O}_{3}}{\mathrm{Al}_{2} \mathrm{O}_{3}}$ & $\frac{\mathrm{Fe}_{2} \mathrm{O}_{3}}{\mathrm{Mn}_{3} \mathrm{O}_{4}}$ & $\mathrm{CaCO}_{3} \mathrm{~b}$ \\
\hline $8-3,62-64$ & 53.38 & 17.27 & 3.72 & 9.20 & 1.36 & 0.81 & 0.06 & 2.83 & 2.72 & 8.64 & 3.09 & 6.76 & 26.79 \\
$10-1,134-139$ & 54.53 & 17.05 & 3.74 & 9.37 & 1.29 & 0.79 & 0.05 & 3.06 & 3.72 & 6.40 & 3.20 & 7.26 & 2.50 \\
$11-2,54-58$ & 55.24 & 17.64 & 3.33 & 7.94 & 1.03 & 0.78 & 0.09 & 3.62 & 4.41 & 5.92 & 3.13 & 7.71 & 9.82 \\
$20-2,115-117$ & 56.05 & 17.28 & 3.97 & 7.53 & 0.72 & 0.86 & 0.16 & 2.84 & 2.10 & 8.49 & 3.24 & 10.46 & 53.93 \\
$22, \mathrm{CC}$ & 56.75 & 16.95 & 5.09 & 6.63 & 0.62 & 1.03 & 0.22 & 2.06 & 0.37 & 10.28 & 3.35 & 10.69 & 86.79 \\
$28-2,62-64$ & 57.17 & 17.15 & 4.15 & 8.58 & 0.83 & 0.88 & 0.08 & 2.55 & 2.48 & 6.13 & 3.33 & 10.34 & 48.39 \\
$36-\mathrm{CC}, 25-26$ & 53.96 & 15.26 & 4.50 & 10.05 & 1.32 & 0.93 & 0.07 & 2.79 & 0.26 & 10.85 & 3.54 & 10.05 & 73.04 \\
$37-3,45-46$ & 51.83 & 15.03 & 3.92 & 11.84 & 2.47 & 0.83 & 0.18 & 3.06 & 3.72 & 7.12 & 3.45 & 11.84 & 19.29 \\
$37, \mathrm{CC}(10-12)$ & 53.44 & 15.47 & 4.16 & 10.40 & 1.80 & 0.87 & 0.07 & 2.73 & 1.47 & 9.58 & 3.45 & 10.40 & 59.11 \\
$44-3,3-5$ & 49.62 & 13.39 & 5.36 & 11.03 & 1.47 & 1.10 & 0.24 & 1.81 & 0.39 & 15.59 & 3.71 & 7.50 & 87.50 \\
$50-2,145-148$ & 48.73 & 11.60 & 5.03 & 8.51 & 1.15 & 1.24 & 0.08 & 1.86 & 0.39 & 21.42 & 4.20 & 7.40 & 86.07 \\
$50-3,25-28$ & 46.63 & 11.89 & 5.94 & 10.06 & 2.89 & 1.01 & 0.18 & 1.74 & 0.46 & 19.21 & 3.92 & 3.48 & 88.93 \\
\hline
\end{tabular}

${ }^{\mathrm{a}} \mathrm{Ign}=$ ignition at $1000^{\circ} \mathrm{C}$

b Calculated from total $\mathrm{CaO}(\%)$ 
Table 15. Trace element chemistry (ppm) of sedimentary deposits, Site 523.

\begin{tabular}{lrrrrrrrrrr}
\hline $\begin{array}{c}\text { Core-Section } \\
\text { (interval in cm) }\end{array}$ & $\mathrm{Sr}$ & $\mathrm{Ba}$ & $\mathrm{V}$ & $\mathrm{Ni}$ & $\mathrm{Co}$ & $\mathrm{Cr}$ & $\mathrm{B}$ & $\mathrm{Zn}$ & $\mathrm{Cu}$ & $\mathrm{Pb}$ \\
\hline $8-3,62-64$ & 779 & 517 & 93 & 196 & 105 & 58 & 112 & 74 & 55 & 16 \\
$10-1,134-139$ & 251 & 537 & 104 & 318 & 119 & 69 & 207 & 90 & 230 & 71 \\
$11-2,54-58$ & 702 & 965 & 116 & 252 & 94 & 65 & 194 & 61 & 142 & 54 \\
$20-2,115-117$ & 1327 & 919 & 69 & 99 & 34 & 26 & 105 & 87 & 55 & 37 \\
$22, \mathrm{CC}$ & 1681 & 437 & 23 & 22 & 6 & $<5$ & $<5$ & $<2$ & 25 & 10 \\
$28-2,62-64$ & 1578 & 477 & 72 & 62 & 24 & 19 & 90 & 71 & 51 & 10 \\
$36, \mathrm{CC}(25-26)$ & 1508 & 217 & 25 & 32 & 17 & $<5$ & 10 & 19 & 45 & 13 \\
$37-3,45-46$ & 770 & 1322 & 127 & 165 & 107 & 42 & 128 & 105 & 81 & 56 \\
$37, \mathrm{CC}(10-12)$ & 978 & 316 & 52 & 84 & 37 & 13 & 56 & 52 & 75 & 46 \\
$44-3,3-5$ & 1438 & 368 & 10 & 11 & 5 & $<5$ & $<5$ & $<2$ & 23 & 6 \\
$50-2,145-148$ & 1154 & 142 & $<5$ & 11 & 4 & $<5$ & $<5$ & $<2$ & 21 & 7 \\
$50-3,25-28$ & 1248 & 168 & 35 & 4 & $<2$ & $<5$ & $<5$ & $<2$ & 33 & 12 \\
& & & & & & & & & &
\end{tabular}

most calcareous sequences are predominantly detrital. The transformed and authigenic smectites increase in the prevalent fine fraction of the iron-rich condensed clay deposits. The relative variation of detrital clays, particularly illite and scarce kaolinite, along the sedimentary columns is too low to suggest that the determining factor is continental climate change through Eocene to Pliocene times. Moreover, the history of the studied sites, their paleobathymetry, and the deep water mass circulation, sea currents, and size of particles involve many factors that have modified the original ratio of the different clay minerals from their continental source. The changes in the origin and in the transportation of detrital compounds are better reflected in the quartz and feldspar proportions at the Pliocene/Miocene and Miocene/Oligocene boundaries. A specific study on feldspar mineralogy might reveal their origin and the relative contribution of continental and basaltic ridge sources.

The variations of the physicochemical characteristics of the water mass modify the deep environment and subsequently the accumulation rate, the sediment interface reactions, and early diagenesis. In the Miocene marly calcareous and clay deposits, early diagenetic changes were determined for the smectites. Such changes, which involve ion exchange reactions, generally occur in areas where sedimentation rate is low, with ample time for chemical interaction between primary clay minerals and seawater. In the South Atlantic, the smectite transformation is related to carbonate dissolution and low sedimentation periods during the Miocene, particularly the upper Miocene (e.g., Sites 519-521). The clay mineral evolution of calcareous oozes to red clay in the $\mathrm{Pa}$ cific Ocean was described by Hoffert (1980).

Within the clayey basal sediments and in places in the sedimentary column, the occurrence of palagonite and volcanic clasts marks the specific ridge influence that induces the authigenesis of smectites such as nontronite and ferrous beidellite; these are morphologically distinct from the transformed smectites of dissolution facies. The authigenesis of volcanogenic smectites is often cited in sediments near oceanic ridges, volcanic seamounts, and from basalt alteration (Hoffert, 1980; Honnorez,

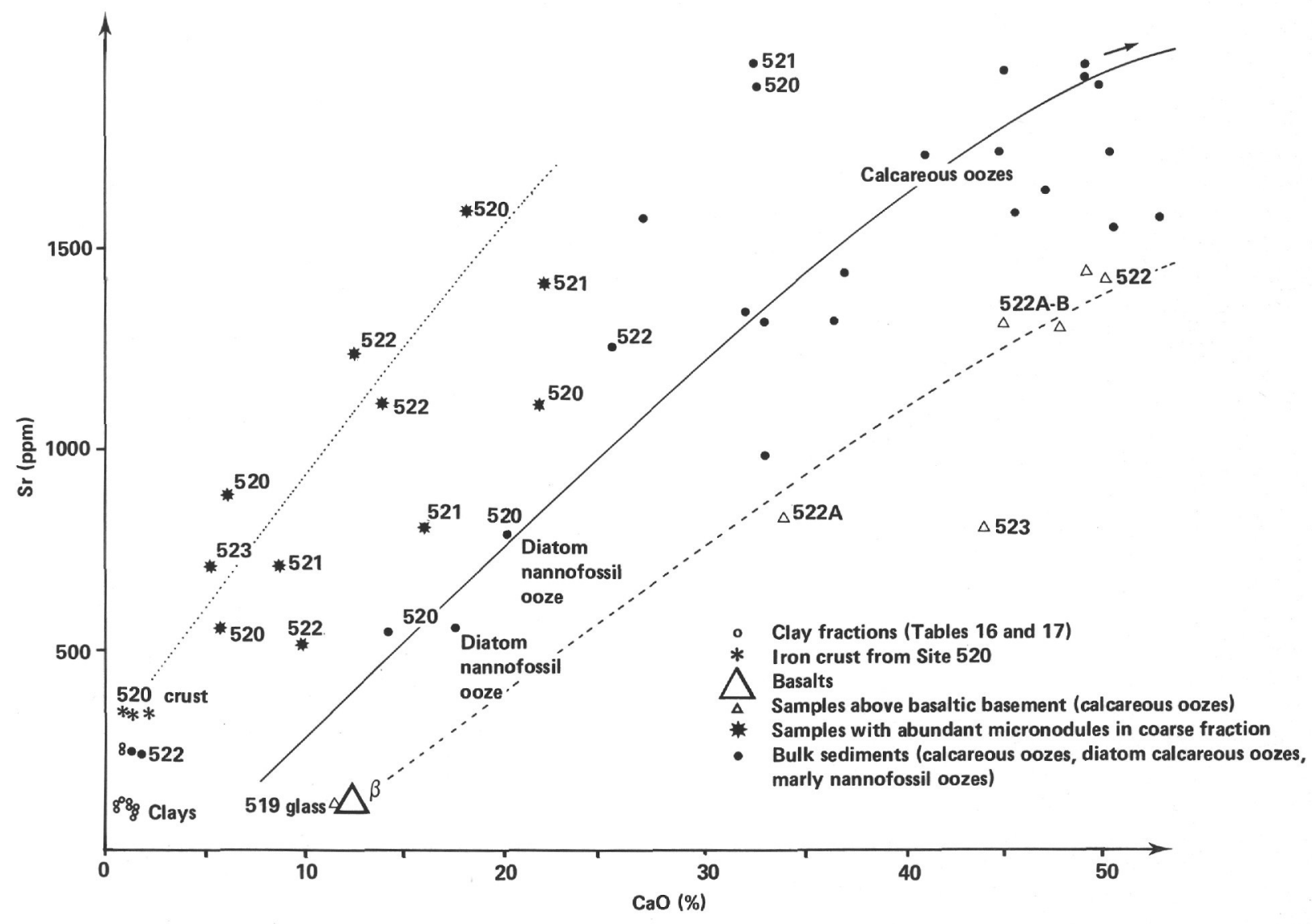

Figure 8. Relationship between $\mathrm{Sr}$ and $\mathrm{CaO}$ contents in sedimentary deposits from Sites 519 to 523 . 
Table 16. Major element chemistry (wt.\%) of clay fraction from some clay deposits at Sites 519 to 523.

\begin{tabular}{|c|c|c|c|c|c|c|c|c|c|c|c|c|}
\hline $\begin{array}{c}\text { Sample } \\
\text { (interval in } \mathrm{cm} \text { ) }\end{array}$ & $\mathrm{SiO}_{2}$ & $\mathrm{Al}_{2} \mathrm{O}_{3}$ & $\mathrm{MgO}$ & $\mathrm{CaO}$ & $\mathrm{Fe}_{2} \mathrm{O}_{3}$ & $\mathrm{Mn}_{3} \mathrm{O}_{4}$ & $\mathrm{TiO}_{2}$ & $\mathrm{Na}_{2} \mathrm{O}$ & $\mathrm{K}_{2} \mathrm{O}$ & $\operatorname{Ign}^{\mathrm{a}}$ & Total & Sediment \\
\hline $519-36-2,20-24$ & 46.5 & 15.7 & 3.05 & 1.0 & 20.8 & 2.49 & 0.80 & 0.53 & 2.98 & 7.08 & 100.94 & \\
\hline $519-36-2,80-84$ & 46.9 & 14.5 & 2.85 & 0.8 & 19.8 & 2.24 & 0.78 & 0.55 & 2.86 & 7.58 & 98.88 & Miocene basal deposits \\
\hline $522 \mathrm{~A}-30-2,43-45$ & 52.5 & 11.8 & 6.45 & 1.6 & 16.0 & 1.67 & 0.84 & 0.42 & 2.87 & 5.57 & 99.74 & \\
\hline $522 \mathrm{~B}-3-2,100-105$ & 49.3 & 13.2 & 3.78 & 1.4 & 16.4 & 4.76 & 0.65 & 0.38 & 3.68 & 7.30 & 100.95 & Eocene basal deposits \\
\hline $520-15-2,38-40$ & 56.3 & 11.5 & 3.21 & 1.1 & 17.1 & 0.34 & 0.60 & 0.50 & 2.28 & 5.67 & 98.68 & \\
\hline $521-11, \mathrm{CC}(15-17)$ & 49.5 & 15.8 & 2.67 & 0.5 & 16.5 & 3.32 & 0.79 & 0.63 & 2.62 & 7.63 & 100.06 & \\
\hline $522-9, \mathrm{CC}$ & 54.3 & 17.1 & 3.08 & 0.6 & 11.6 & 2.25 & 0.74 & 0.65 & 3.14 & 6.68 & 100.21 & Miocene red clay \\
\hline $522-11-2,114-116$ & 53.1 & 17.4 & 3.70 & 0.8 & 10.2 & 1.75 & 0.72 & 0.90 & 3.84 & 6.25 & 98.80 & \\
\hline $522-11, \mathrm{CC}(10-12)$ & 52.6 & 17.5 & 3.79 & 0.9 & 10.4 & 1.73 & 0.70 & 0.97 & 3.83 & 6.70 & 99.19 & \\
\hline $523-47-1,120-122$ & 58.7 & 13.4 & 3.27 & 1.2 & 9.9 & 1.48 & 0.75 & 0.47 & 3.31 & 6.16 & 98.91 & Eocene red clay \\
\hline
\end{tabular}

Note: Total iron is calculated as $\mathrm{Fe}_{2} \mathrm{O}_{3}$ and total manganese as $\mathrm{Mn}_{3} \mathrm{O}_{4}$.

$\mathrm{a}_{\text {Ign }}=$ ignition at $1000^{\circ} \mathrm{C}$

Table 17. Trace element chemistry (ppm) of clay fraction from some clay deposits at Sites 519 to 523 .

\begin{tabular}{lrrrrrrrr}
\hline $\begin{array}{c}\text { Sample } \\
\text { (interval in cm) }\end{array}$ & \multicolumn{1}{c}{$\mathrm{Sr}$} & $\mathrm{Ba}$ & $\mathrm{V}$ & $\mathrm{Ni}$ & $\mathrm{Co}$ & $\mathrm{Cr}$ & $\mathrm{Zn}$ & $\mathrm{Cu}$ \\
\hline $519-36-2,20-24$ & 118 & 302 & 362 & 166 & 112 & 54 & 214 & 262 \\
$519-36-2,80-84$ & 119 & 304 & 348 & 166 & 119 & 54 & 215 & 250 \\
$522-30 \mathrm{~A}-2,43-45$ & 77 & 401 & 162 & 140 & 78 & 74 & 347 & 308 \\
$522-3 \mathrm{~B}-2,100-105$ & 117 & 1134 & 295 & 275 & 86 & 52 & 268 & 205 \\
$520-15-2,38-40$ & 101 & 189 & 214 & 180 & 80 & 54 & 184 & 119 \\
$521-11, \mathrm{CC}(15-17)$ & 97 & 355 & 281 & 277 & 242 & 71 & 177 & 225 \\
$522-9, \mathrm{CC}$ & 108 & 398 & 172 & 289 & 191 & 77 & 170 & 196 \\
$522-11-2,114-116$ & 251 & 382 & 159 & 256 & 135 & 66 & 182 & 172 \\
$522-11, \mathrm{CC}, 10-12$ & 249 & 336 & 153 & 242 & 133 & 66 & 202 & 156 \\
$523-47-1,120-122$ & 155 & 1735 & 117 & 111 & 57 & 41 & 183 & 113 \\
\hline
\end{tabular}

1981; McMurtry and Yeh, 1981). In the Atlantic Ocean most of the studies were made in the FAMOUS (FrancoAmerican Mid-Ocean Undersea Study) area and for the North Mid-Atlantic Ridge.

\section{Oxides}

Another characteristic of the dissolution facies from Miocene calcareous oozes is the early formation of metalliferous oxides as colloids, granules, or microconcretions. Iron oxides, which are strongly associated with clays, increase with the degree of dissolution of the sediments. On the residual foraminiferal tests, one of the first effects of the cooling of deep water is the precipitation of manganese oxides. In the clay-rich deposits, the coarse fraction contains prevalent micronodules. In both cases the concentration of iron oxides and the formation of Mn-micronodules are related to simultaneous dissolution processes and cooling in an oxidized environment. The development of a condensed red clay facies was inhibited at Site 520, which has a different localized environment (a silled less oxic basin with slumps) that permitted the preservation of siliceous biogenic phases. These deposits show particularly well crystallized manganese oxides.

The sporadic volcanic manifestations through the sedimentary column promote the formation of a siliceous iron crust over volcaniclastic compounds.

The basal pelagic sediments comprise micronodules and ferromagnanese oxides which cover in-place palagonite and volcanic fragments. The genesis of ironmanganese compounds near mid-oceanic ridges is often described and attributed to the formation of metalliferous sediments (Toth, 1980).

\section{CONCLUSION}

The red clay facies found at Drill Sites 519 to 523 along a transect in the South Atlantic results from two closely associated oceanographic influences:

1) The dissolution of calcareous oozes is related to the CCD rise and later a low sedimentation rate, a change in the water mass circulation, and a cooling event in the middle and late Miocene. The biogenic and geochemical consequences of the cooling are described by Haq $(1980,1981)$ and related to these sedimentary sections by McKenzie et al. (this vol.). These dissolution processes involve the transformation of clay minerals and the early authigenesis of $\mathrm{Fe}$-oxides and $\mathrm{Mn}$-microconcretions, with specific concentrations of $\mathrm{Ni}$ and $\mathrm{Sr}$.

2) The basalt alteration and volcanic manifestations are marked in basal sediments and in some layers. These influences promote the authigenesis of smectites, manganese concretions, and siliceous iron crust; they are chemically characterized by $\mathrm{Mg}, \mathrm{Ti}, \mathrm{V}$, and $\mathrm{Cr}$ contents.

The convergence of both evolutions ended in the formation of red clay at the sites nearest the ridge crest (Sites 519 and 521). The red clay layers and marly oozes from the Eocene and Miocene sequences are more differentiated in the sites farther from the Mid-Atlantic Ridge crest (Sites 522 and 523).

\section{ACKNOWLEDGMENTS}

The analytical work was performed with the technical support of the Centre de Sedimentologie et Geochimie de la Surface and of the Institut de Geologie de Strasbourg. This research has also been reported by the C.N.R.S., France (A.T.P.-G.G.O. No. 1681).

I am grateful to J. Coves, P. Gruner, A. Pimmel, C. Romon, and J. P. Schmitt for helpful technical contributions. T.E.M. studies were made with the collaboration of G. Ehret. I also want to thank A. Schaaf and B. Allenbach for a friendly presence, J. A. McKenzie for our discussions, and K. A. Pisciotto for his technical and stylistic revisions.

\section{REFERENCES}

Arrhenius, G., 1963. Pelagic sediments. In Hill, M. N. (Ed.), The Sea: New York (Interscience), 3:655-718.

Besnus, Y., and Rouault, R., 1973. Une methode d'analyse des roches au spectrometre d'arc a lecture directe par un dispositif d'electrode rotative. Analusis, 2:111-116.

Borst, R. L., and Keller, W. D., 1969. Scanning electron micrographs of API references clay minerals and other selected samples. Proc. Int. Clay Conf., Tokyo, 871-901.

Boyle, E. A., 1981. Cadmium, zinc, copper and barium in foraminiferal test. Earth Planet. Sci. Lett., 53:11-35.

Chow, T. J., and Goldberg, E. D., 1970. On marine geochemistry of barium. Geochim. Cosmochim. Acta, 39:1579-1582. 
Chukhrov, F. V., Gorshkov, A. I., Rudnitskaya, E. S., Beresovskaya, V. V., and Sivtsov, A. V., 1980. Manganese minerals in clays: a review. Clays Clay Miner., 28:346-354.

El Wakeel, S. K., and Riley, J. P., 1961. Chemical and mineralogical studies of deep sea sediments. Geochim. Cosmochim. Acta, 25 $110-146$.

Glasby, G. P., 1977. Marine Manganese Deposits: Amsterdam (Elsevier), 15:523.

1978. Deep sea manganese nodules in the stratigraphic record-evidence from DSDP cores. Mar. Geol., 28:51-64.

Hajash, A., 1975. Hydrothermal process along Mid-Ocean Ridges: an experimental investigation. Contrib. Mineral. Petrol., 53:205-226.

Haq, B. U., 1980. Biogeographic history of Miocene calcareous nannoplankton and paleoceanography of the Atlantic Ocean. Micropaleontology, 26:414-443.

1981. Paleogene paleoceanography. Early Cenozoic oceans revisited. Oceanol. Acta, 80:71-82.

Hoffert, M., 1980. Les “Argiles Rouges des Grands Fonds" dans le Pacifique Centre-Est. Authigenese, transport, diagenese. Sci. Géol. Mém., 61:231.

Hoffert, M., Karpoff, A. M., Clauer, N., Shaaf, A., Courtois, C., and Pautot, G., 1978. Néoformations et altérations dans trois faciès volcanosédimentaires du Pacifique Sud. Oceanol. Acta, 1: 187-202.

Honnorez, J., 1978. Generation of phillipsite by palagonitization of basaltic glass in sea water and the origin of K-rich deep-sea sediments. In Sand, L. B., and Mumpton, F. A. (Eds.), Natural Zeolites: New York (Pergamon Press), pp. 245-258.

, 1981. The aging of the oceanic crust at low-temperature. In Hill, M. N. (Ed.), The Sea: New York (Interscience), Vol. 7.

Karpoff, A. M., 1980. The sedimentary deposits of Suiko Seamount (Leg 55, Site 433): from the reef environment to the pelagic sedimentation. In Jackson, E. D., Koizumi, I., et al., Init. Repts. DSDP, 55: Washington (U.S. Govt. Printing Office), 491-501.

Karpoff, A. M., Hoffert, M., and Clauer, N., 1981. Sedimentary sequences at Deep Sea Drilling Project Site 464: silicification processes and transition between siliceous biogenic oozes and brown clays. In Thiede, J., Vallier, T. L., et al., Init. Repts. DSDP, 62: Washington (U.S. Govt. Printing Office), 759-771.

Karpoff, A. M., Peterschmitt, I., and Hoffert, M., 1980. Mineralogy and geochemistry of sedimentary deposits on Emperor Seamounts, Sites 430, 431, and 432: authigenesis of silicates, phosphates, and ferromanganese oxides. In Jackson, E. D., Koizumi, I., et al., Init. Repts. DSDP, 55: Washington (U.S. Govt. Printing Office), 463-489.

Kastner, M., and Stonecipher, S. A., 1978. Zeolites in pelagic sediments of Atlantic, Pacific and Indian Oceans. In Sand, L. B., and
Mumpton, F. A., (Eds.), Natural Zeolites: New York (Pergamon Press), pp. 199-220.

Khoury, H. N., and Eberl, D. D., 1979. Bubble-wale shards altered to montmorillonite. Clays Clay Miner., 27:291-292.

Kohyama, N., and Sudo, T., 1975. Hisingerite occurring as a weathering product of iron-rich saponite. Clays Clay Miner., 23: 215-218.

La genese des nodules de manganese, 1979. Colloq. Int. C.N.R.S., 289:410.

McMurtry, G. M., and Yeh, H.-W., 1981. Hydrothermal clay mineral formation of East Pacific Rise and Bauer Basin sediments. Chem. Geol., 32:189-205.

Melson, W. G., and Thompson, G. 1973. Glassy abyssal basalts, Atlantic sea floor near St. Paul's rocks: petrography and composition of secondary minerals. Geol. Soc. Am. Bull., 84:703-716.

Mise au point collective, 1975. Techniques de preparation des mineraux argileux en vue de l'analyse par diffraction des rayons-X. Notes Tech. Inst. Géol., Universite Louis Pasteur, Strasbourg, 1: 21.

Parfitt, R. L., and Hemni, T., 1980. Structure of some allophanes from New Zealand. Clays Clay Miner., 28:285-294.

Schwertmann, U., and Taylor, R. M., 1977. Iron oxides. In Dixon, J. B., and Weed, S. B., (Eds.), Minerals in Soil Environments. SSSA Spec. Publ., pp. 145-180.

1979. Natural and synthetic poorly crystallized lepidocrocite. Clay Miner., 14:185-194.

Sheppard, R. A., and Gude, A. J., 1969. Diagenesis of tuffs in the Barstow formation, Mud Hill, San Bernardino Country, California. Geol. Surv. Prof. Pap. U.S., 634:34.

Sorem, R. K., and Fewkes, R. H., 1977. Internal characteristics. In Glasby, G. P. (Ed.), Marine Manganese Deposits: Amsterdam (Elsevier), 15:147-184.

Toth, J. R., 1980. Deposition of submarine crusts rich in manganese and iron. Geol. Soc. Am. Bull., 91:44-54.

Trauth, D., Ehret, G., Eberhart, J. P., and Weber, F., 1977. Microscopie électronique et minéraux argileux: résultats obtenus et orientations actuelles. Notes Tech. Inst. Geol., Université Louis Pasteur, Strasbourg, 7:16.

Turekian, K. K., 1964. The marine geochemistry of strontium. Geochim. Cosmochim. Acta, 28:1479-1496.

Violante, P., and Tait, J. M., 1978. Identification of imogolite in some volcanic soils from Italy. Clay Miner., 14:155-158.

Date of Initial Receipt: September 1, 1982 


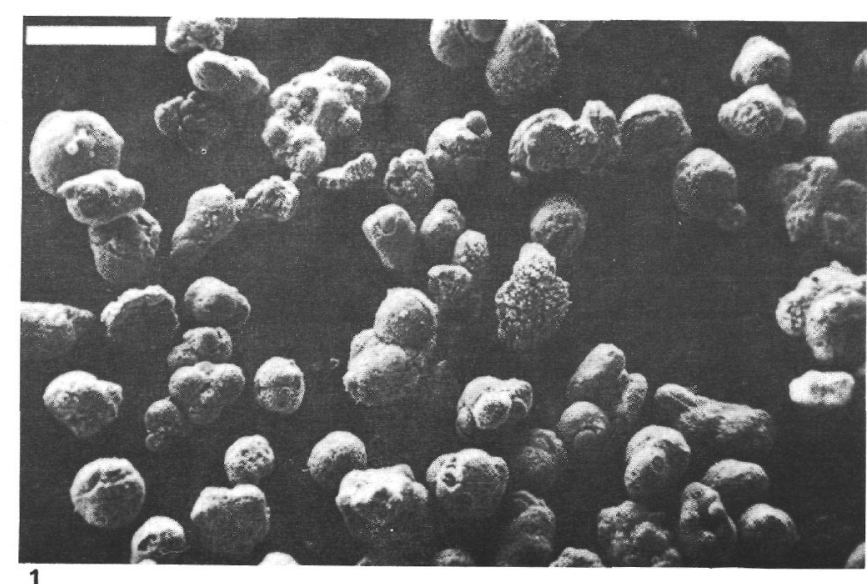

1
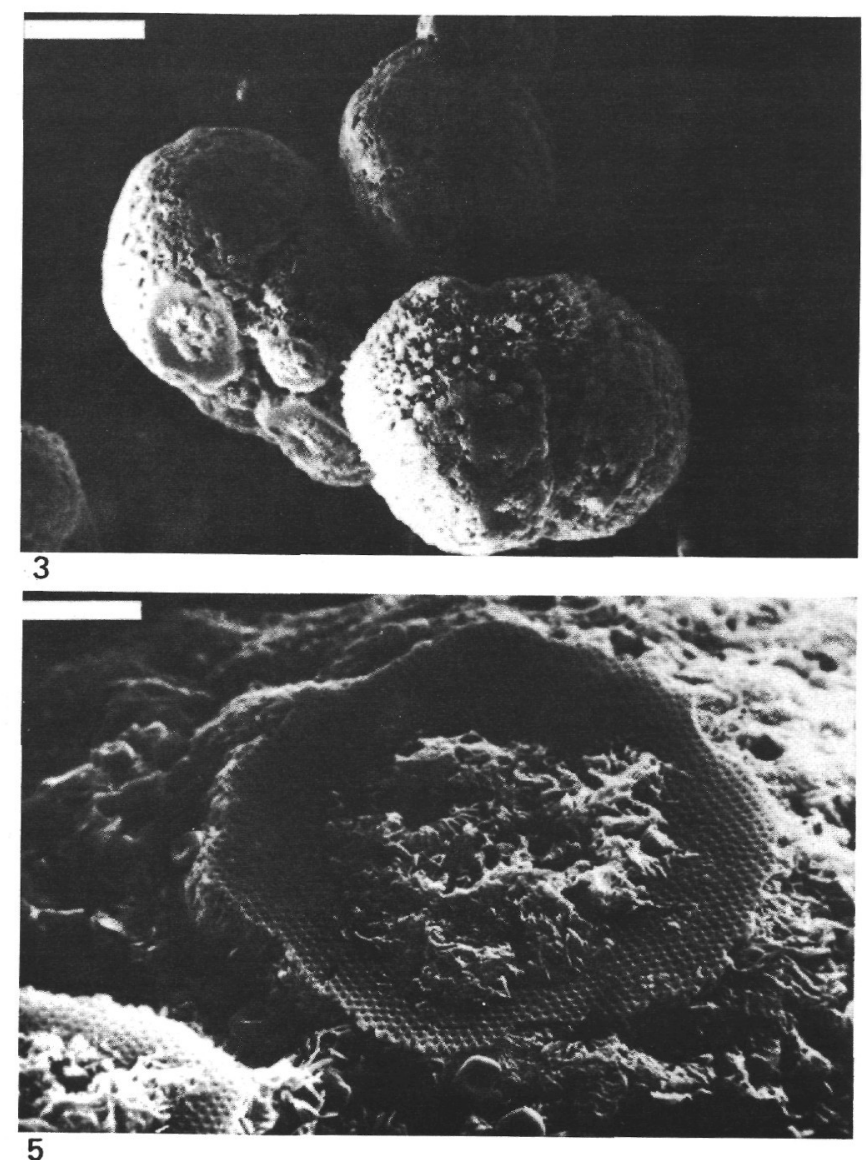

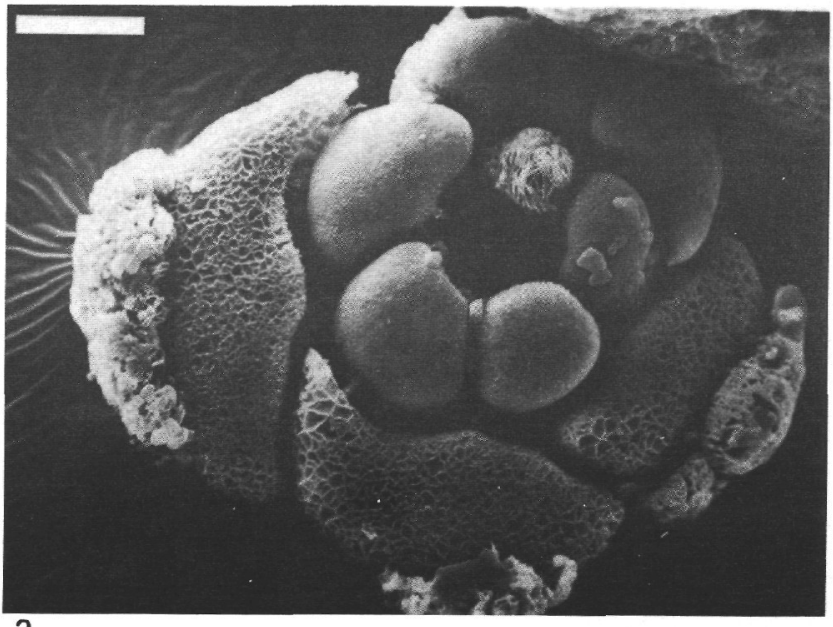

2

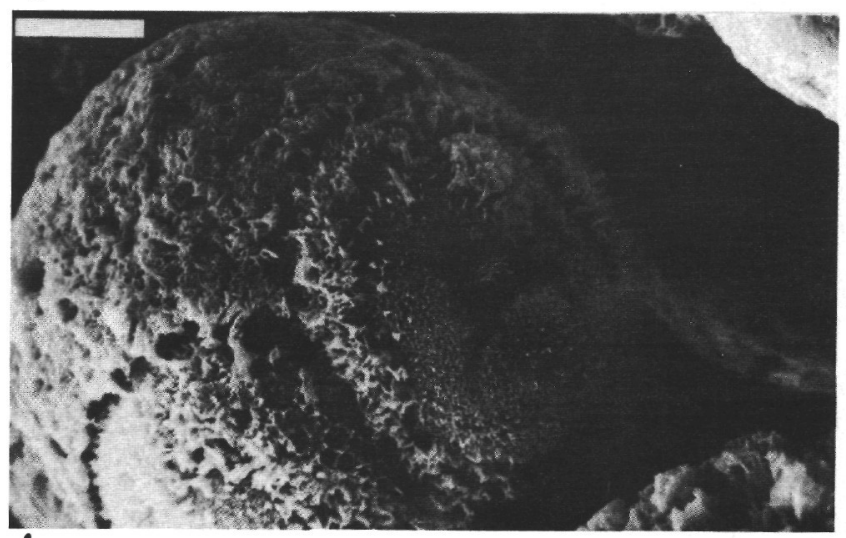

4

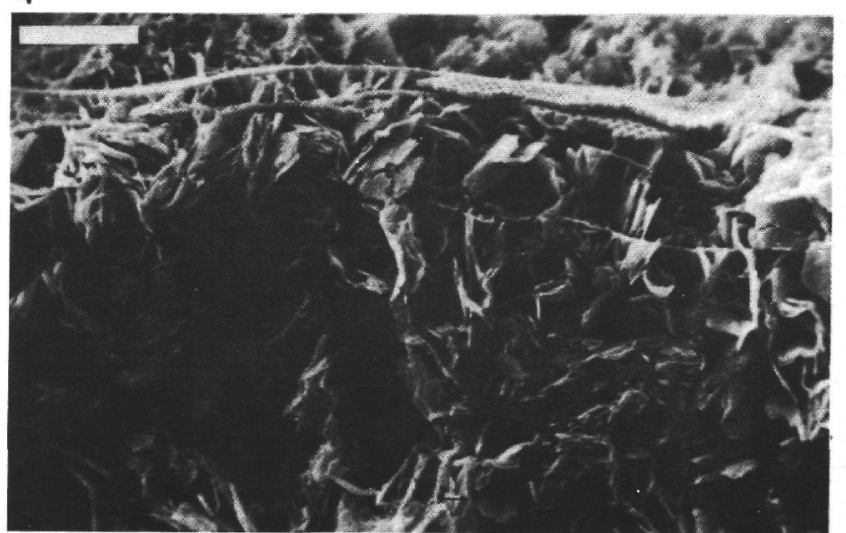

6

Plate 1. SEM photomicrographs of micronodules in the coarse fraction from Miocene diatom nannofossil ooze. Sample 520-16-4, 55-57 cm. 1. General view of the coarse fraction micronodules. Scale bar $=200 \mu \mathrm{m}$. 2. Micronodule. Detail of (1) showing foraminiferal shape without calcareous test. The internal mold is made of manganese oxides of various crystallinities. Scale bar $=20 \mu \mathrm{m}$. 3. Rounded micronodules with diatom fragments coated by manganese oxides. Scale bar $=50 \mu \mathrm{m}$. 4. Detail of a rounded micronodule with successive layers of well crystallized manganese oxides showing the internal mold left by a foraminifer. Scale bar $=20 \mu \mathrm{m}$. 5. Diatom fragment and manganese crystals on the surface of a micronodule. Small cubes in lower part of picture are secondary halite crystals (drying effect). Scale bar $=10 \mu \mathrm{m} .6 .6$. Detail of the relationship of diatom fragment and upright-growing manganese oxide crystals. Scale bar $=5 \mu \mathrm{m}$. 

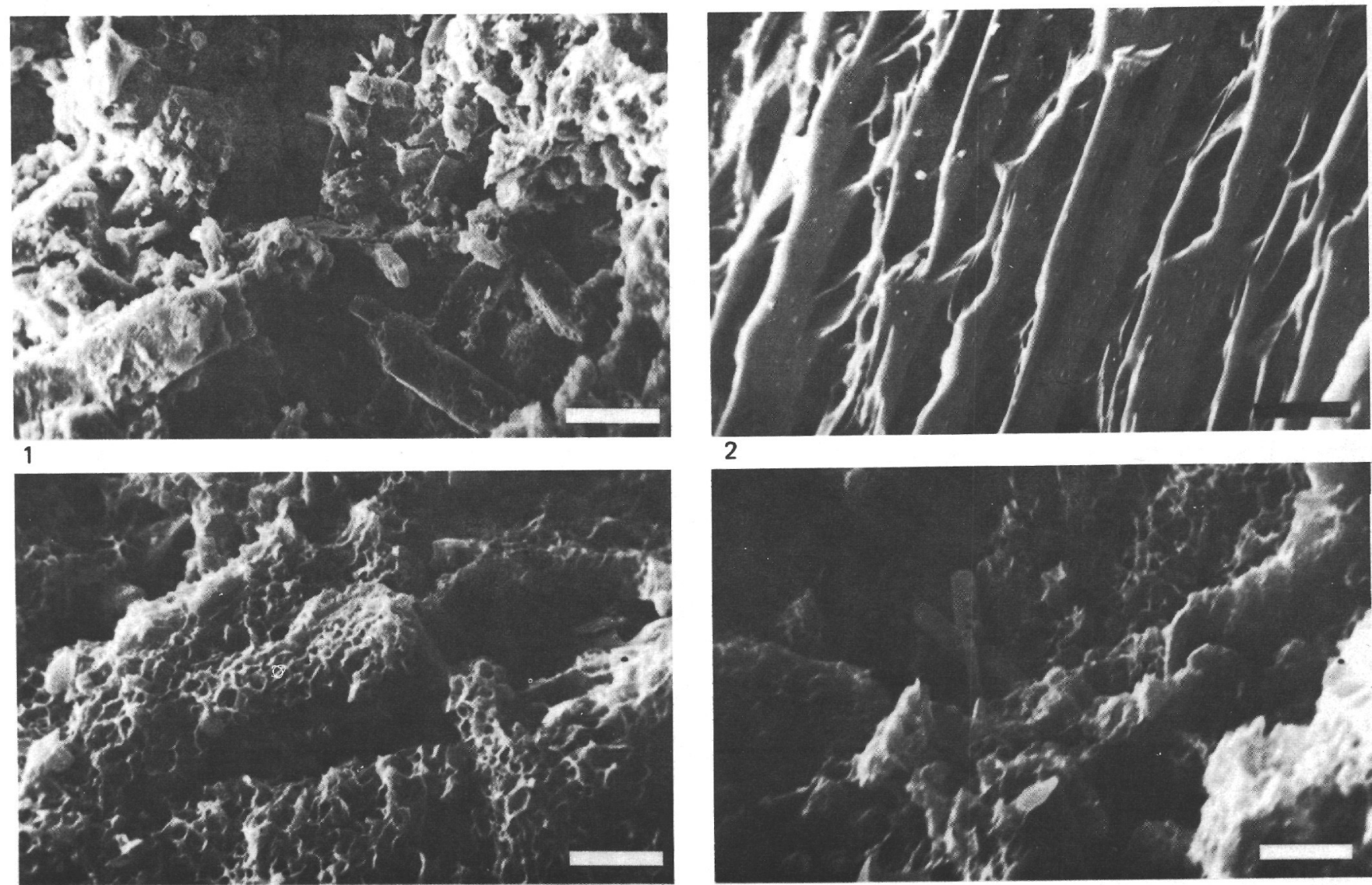

3
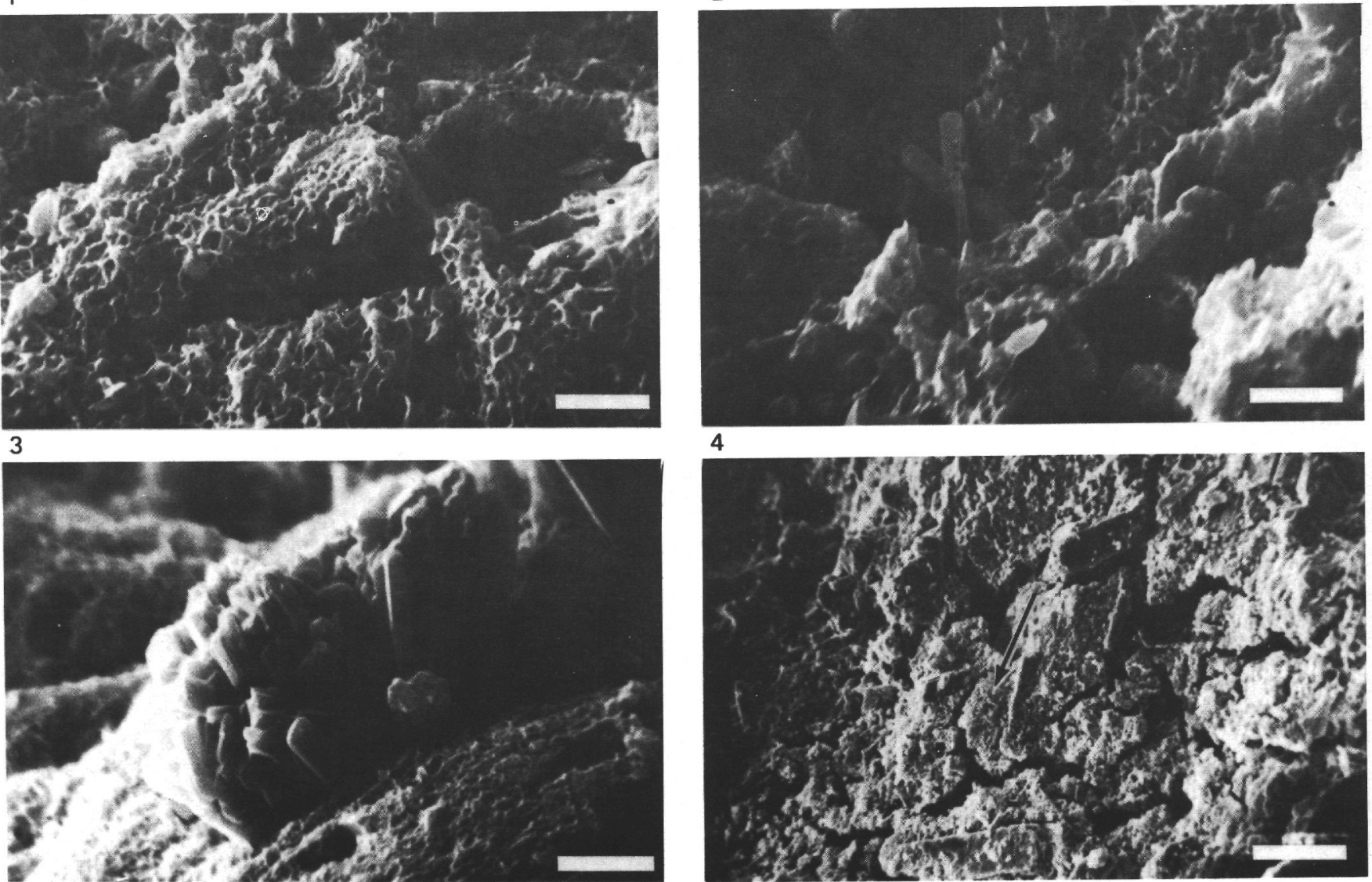

5

4

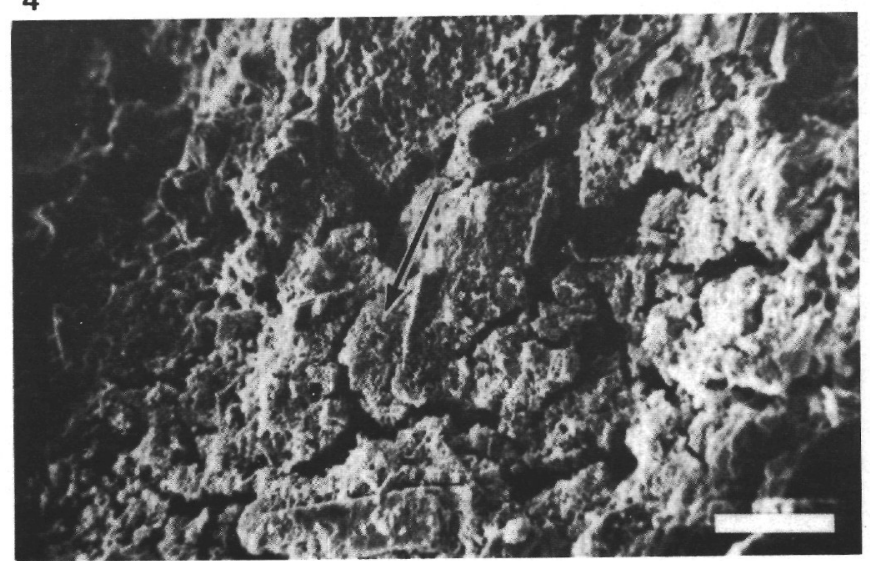

6

Plate 2. SEM microphotographs showing successive authigenesis of silicates and oxides in volcaniclastic particles from the coarse fraction of Pliocene foraminifer nannofossil ooze. Sample 521-7-5,74-75 cm. 1. General view of the particle: aggregate of large zeolites and clays overlying volcanic glass fragment. Scale bar $=20 \mu \mathrm{m}$. 2. Detail of the surface of an altered volcanic glass fragment visible in the coarse particle. Scale $\mathrm{bar}=10 \mu \mathrm{m}$. 3. Authigenic smectites coating the large primary zeolite. Scale bar $=5 \mu \mathrm{m}$. 4. Small secondary twinned zeolite crystals over the authigenic smectites. Scale bar $=5 \mu \mathrm{m}$. 5. Bunch of small zeolites (second generation). Scale bar $=5 \mu \mathrm{m}$. 6. Massive manganese oxides coating and immersing the clays and zeolites (at arrow). Scale bar $=50 \mu \mathrm{m}$. 

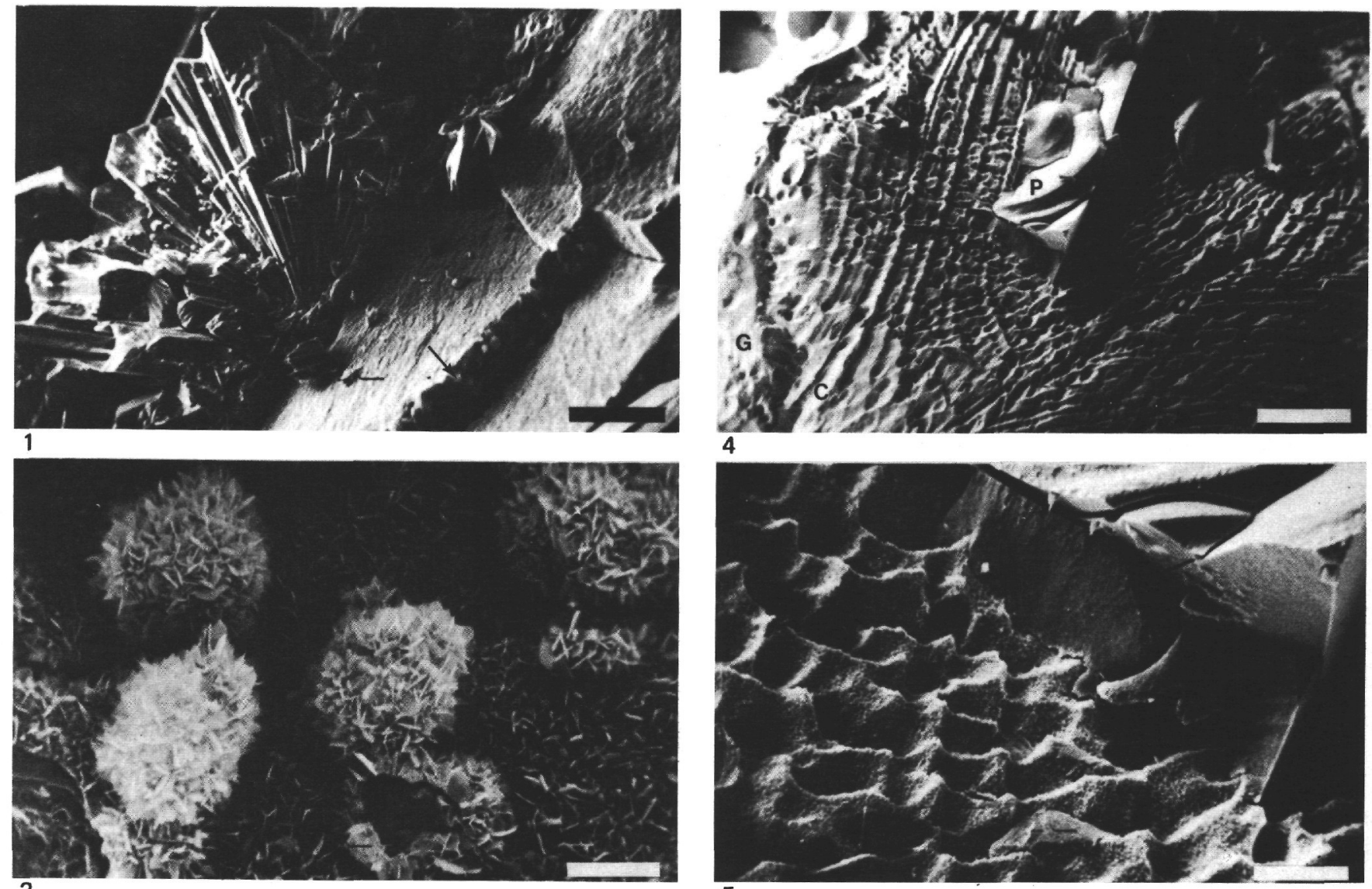

4

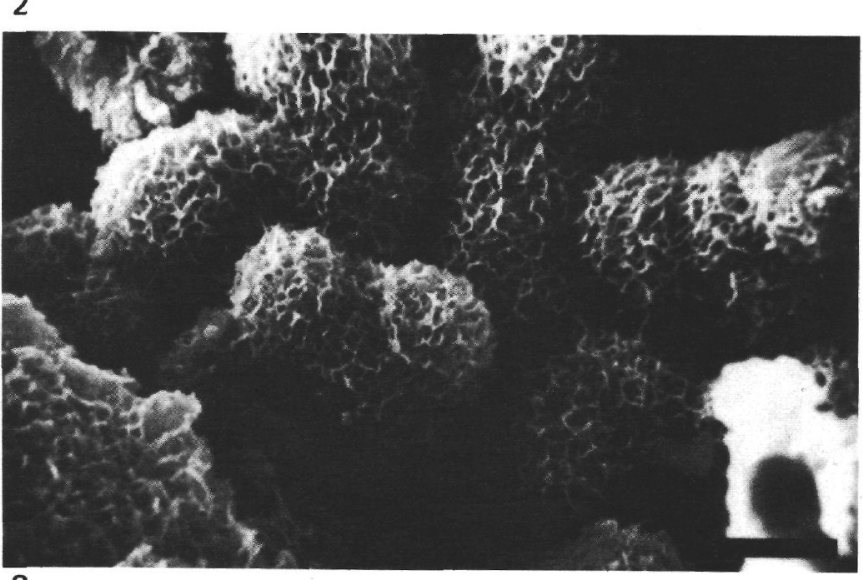

3
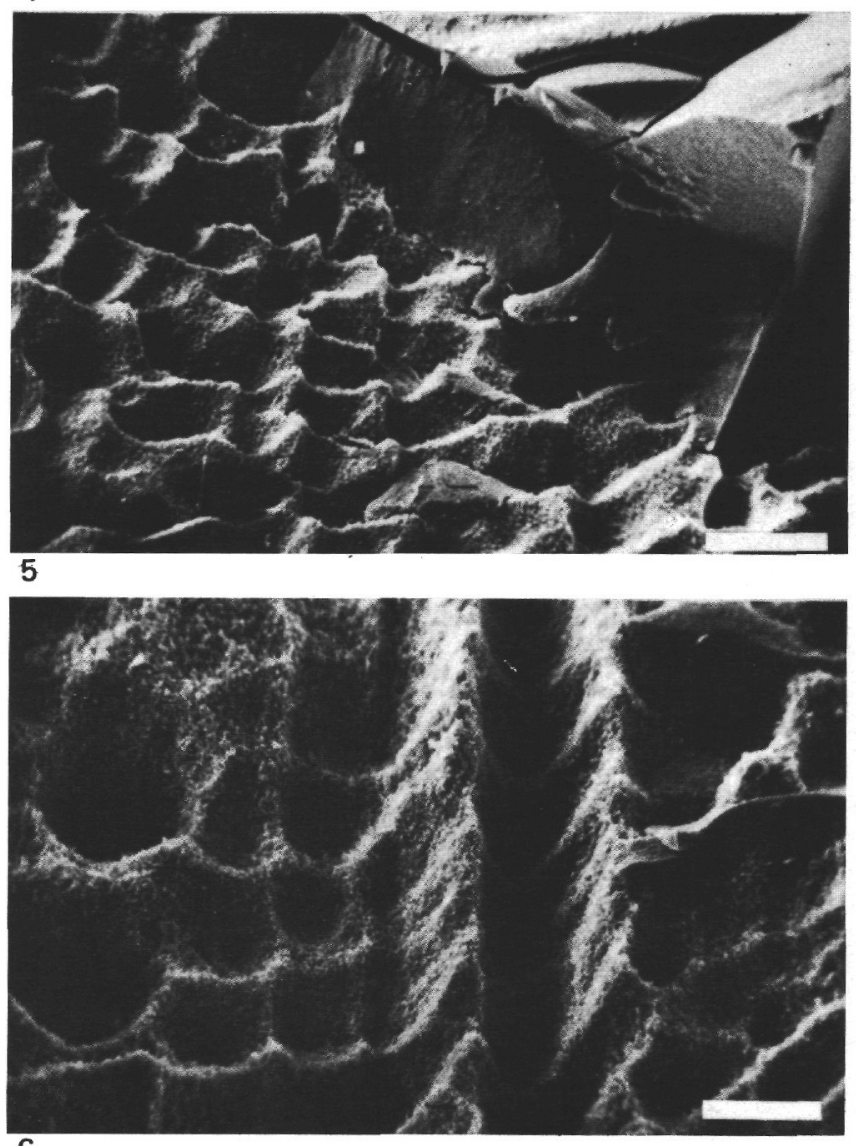

Plate 3. SEM microphotographs showing first weathering products of basaltic basement in altered volcanic breccia from basalt flow Unit 2 at Site 519. Sample 519A-8-4, 139-144 cm (Piece 8). 1. Bundle of phillipsite crystals over palagonite and crystallization of oxides filling small gash (at arrow). Scale bar $=100 \mu \mathrm{m}$. 2. Detail of well crystallized manganese oxides in palagonite gashes. Scale bar $=5 \mu \mathrm{m}$. 3. Local filling of bushshaped authigenic clays in small pores of palagonite. Scale bar $=10 \mu \mathrm{m}$. 4. Rounded glass $(\mathrm{G})$ and palagonite $(\mathrm{P})$ fragment composing the volcanic breccia. A fine layer of clay appears between the glass and palagonite (C). Scale bar $=0.5 \mu \mathrm{m}$. 5. Detail of the wave-shaped clay layer below palagonite. Scale bar $=100 \mu \mathrm{m}$. 6. Detail of the fine-grained surface of the clay. Scale bar $=50 \mu \mathrm{m}$. 
1
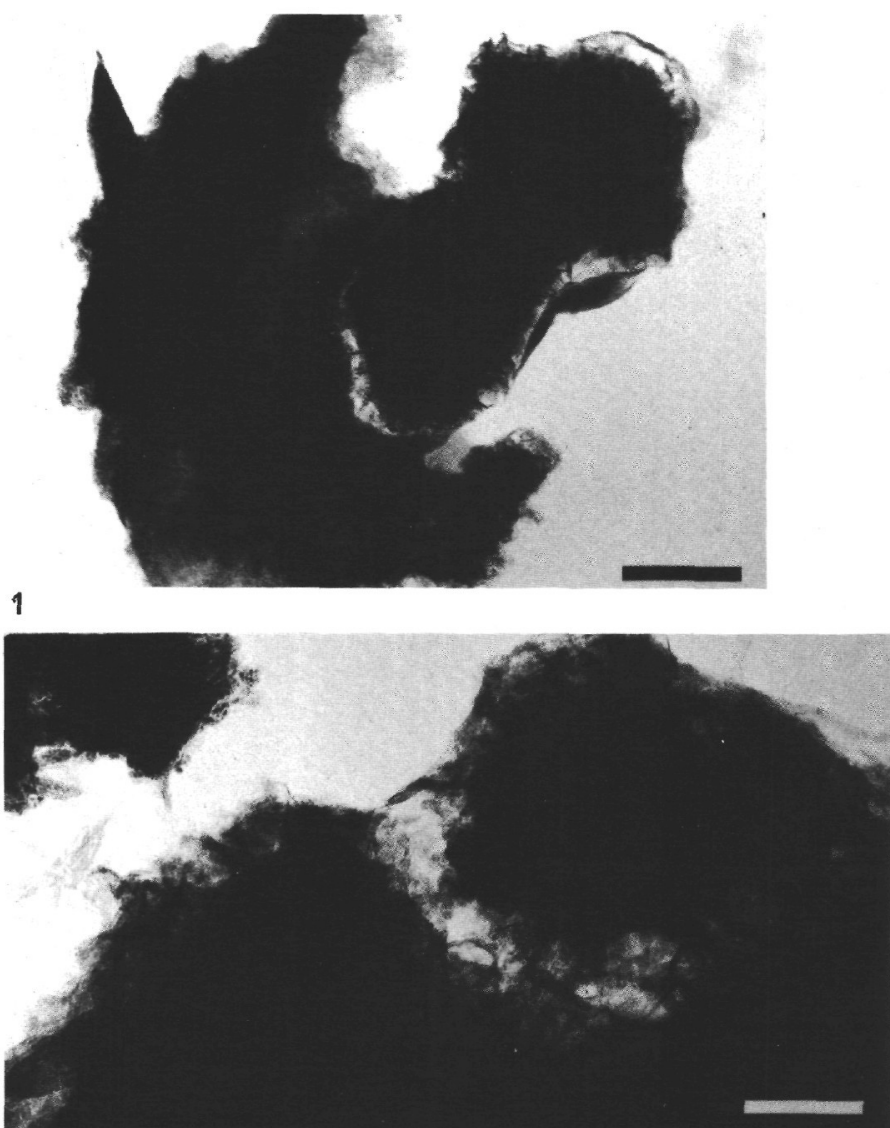

3

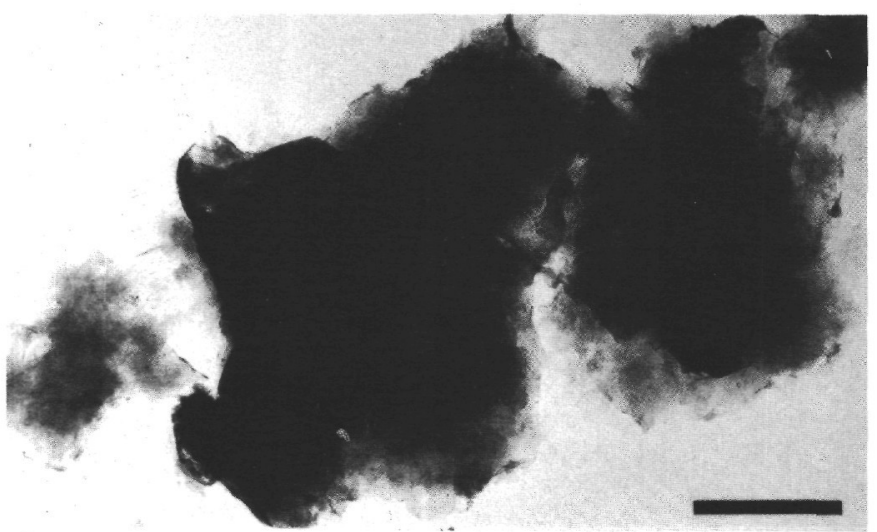

5

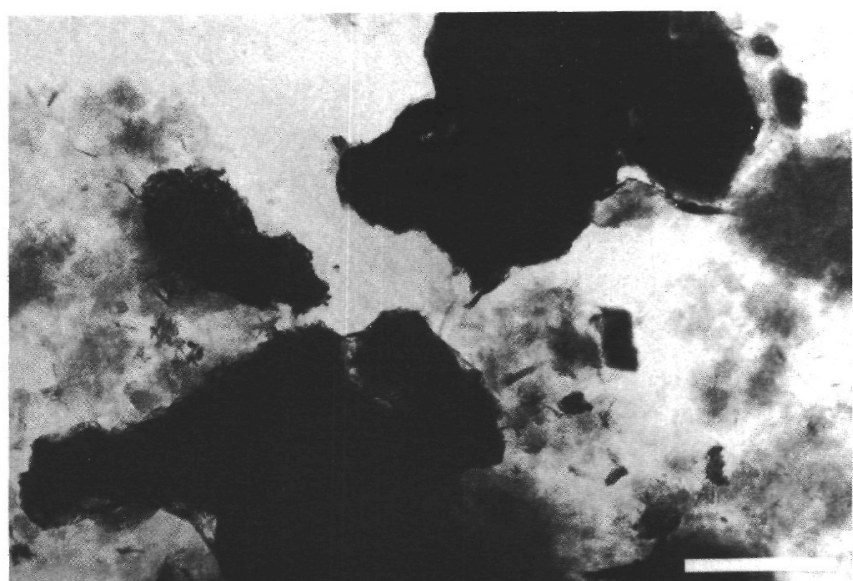

2

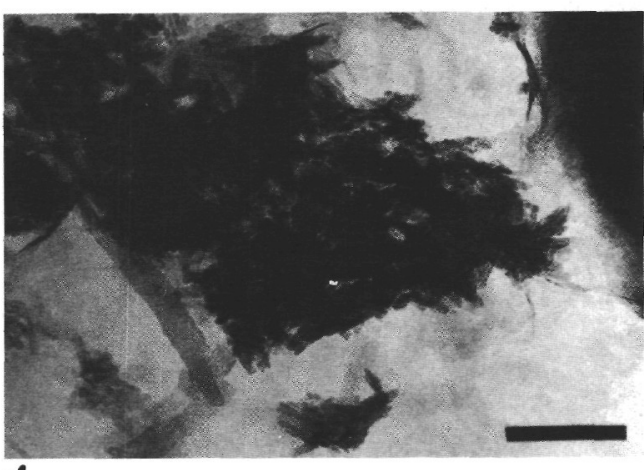

4

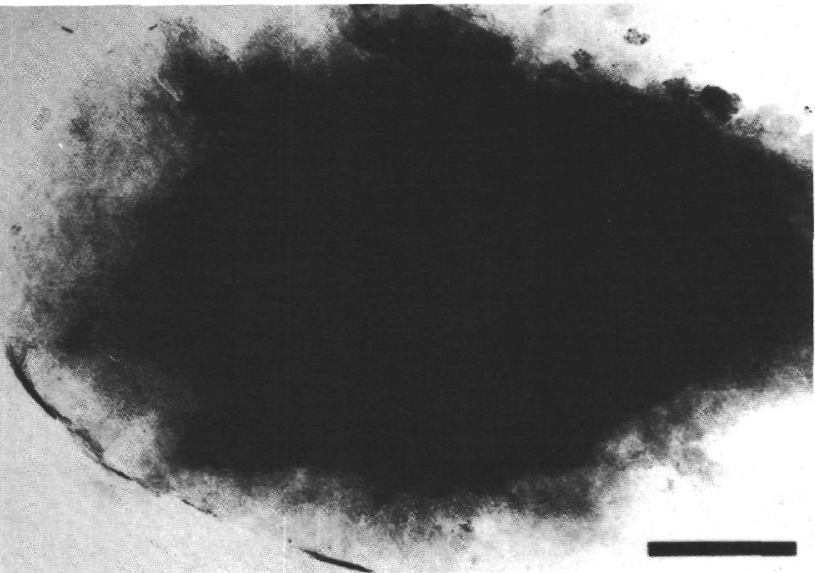

6

Plate 4. TEM microphotographs showing the contribution of basaltic basement alteration to the authigenesis of clays. Photographs are of the clay fraction from marly nannofossil ooze at Sites 519 (Miocene) and 522 (Eocene). 1. Morphology of smectite particles with hairy outlines. Sample 519-36-2, 80-84 cm. Scale bar $=0.2 \mu \mathrm{m}$. 2. Morphology of smectites similar to those in (1). Large particle with curled edges and small particles with hairy outlines. Sample 522B-3-2, 100-102 cm. Scale bar $=0.5 \mu \mathrm{m}$. 3. Detail of the smectites with hairy edges. These smectites are similar to authigenic iron-rich smectites. Sample 522B-3-2, 100-102 cm. Scale bar $=0.1 \mu \mathrm{m}$. 4. Detail of clay aggregate of small bent laths, similar to an iron-oxide/layer-silicate complex; scarce lath-shaped particles are similar to palygorskite. Sample 522B-3-2, 100-102 cm. Scale bar $=0.1 \mu \mathrm{m}$. 5. Large smectite particles with curled overgrowths. These smectites are typical of those from dissolution facies of calcareous oozes. Sample 519-36-2, 80-84 cm. Scale bar $=0.5 \mu \mathrm{m}$. 6. Large smectites with lath-shaped edges and curled overgrowths. Photograph shows transformation of detrital smectites in dissolution facies of calcareous oozes. Sample 522A-30-2, 43-45 cm. Scale bar $=0.5 \mu \mathrm{m}$. 


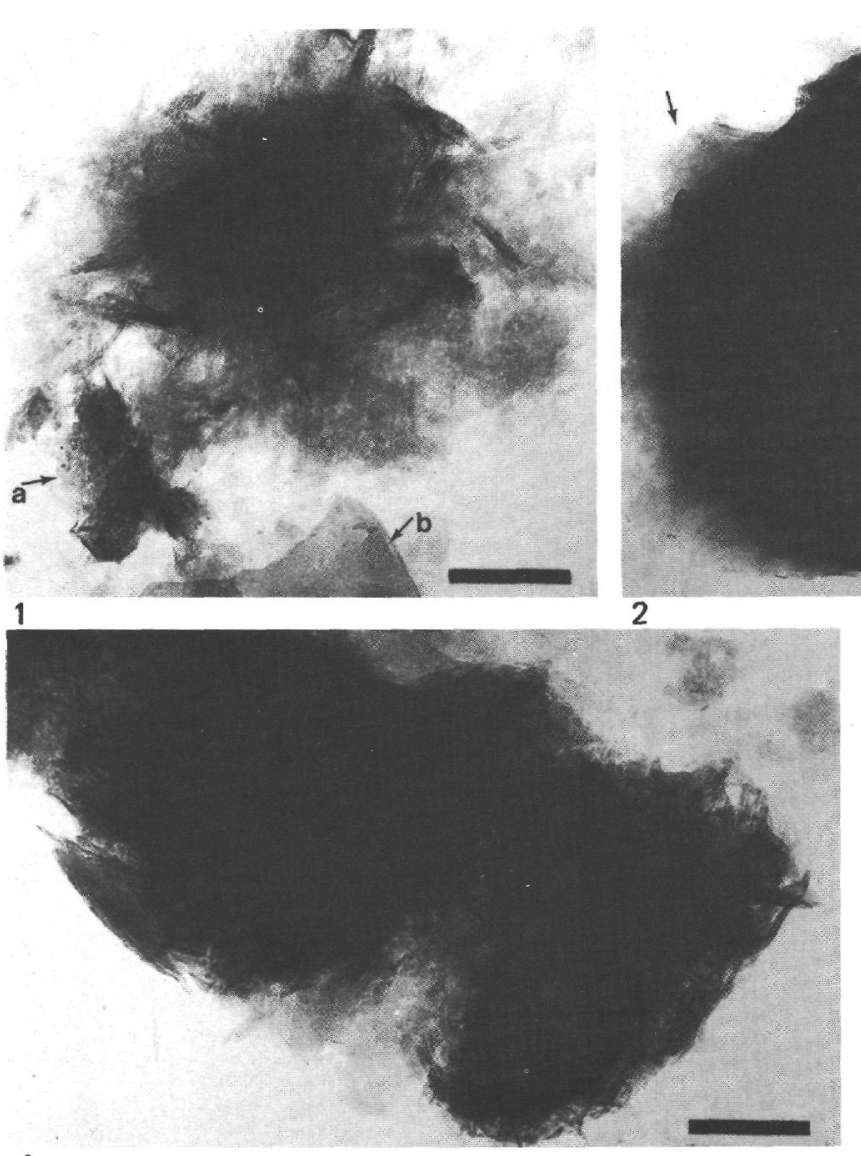

4

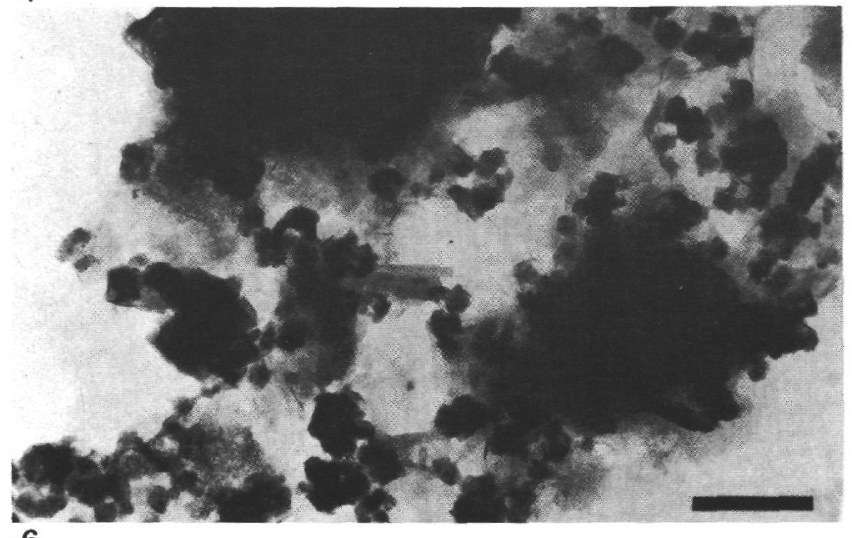

6

Plate 5. TEM microphotographs showing different morphologies of smectites and oxides. Photographs are of clay fractions from Miocene nannofossil clay and clay at Sites 521 and 522. 1. Typical morphology of smectite in reds clay due to dissolution of calcareous ooze. The particles have light outlines with fine laths. Sediment contains small granular clay-iron-oxide flakes (a) and fragments of detrital clays (b). Sample 521$11, C C(15-17 \mathrm{~cm})$. Scale bar $=0.16 \mu \mathrm{m}$. 2. Large smectite particle with curled outlines and scarce overgrowths of laths (at arrow). Sample 5229,CC. Scale bar $=0.3 \mu \mathrm{m}$. 3. Primary well crystallized smectite. Sample 522-9,CC. Scale bar $=0.1 \mu \mathrm{m}$. 4. Smectite particle with hairy outlines as overgrowths of authigenic smectite. Sample 521-11,CC $(15-17 \mathrm{~cm})$. Scale bar $=0.1 \mu \mathrm{m}$. 5. Smectite with small granular iron oxides over the sheets. Detrital clay appears in top left corner. Sample 522-11-2, 114-116 cm. Scale bar $=0.3 \mu \mathrm{m}$. 6. Smectite particles with light overgrowths and flaky aggregates of iron oxides connected by veils or membranes. Sample 522-33,CC $(10-18 \mathrm{~cm})$. Scale bar $=0.2 \mu \mathrm{m}$. 7 . Detail of smectite particles, rounded aggregates (oxides), and fine veils similar to those of some allophanes. Sample $522-33, \mathrm{CC}(10-18 \mathrm{~cm})$. Scale bar = $0.4 \mu \mathrm{m}$. 

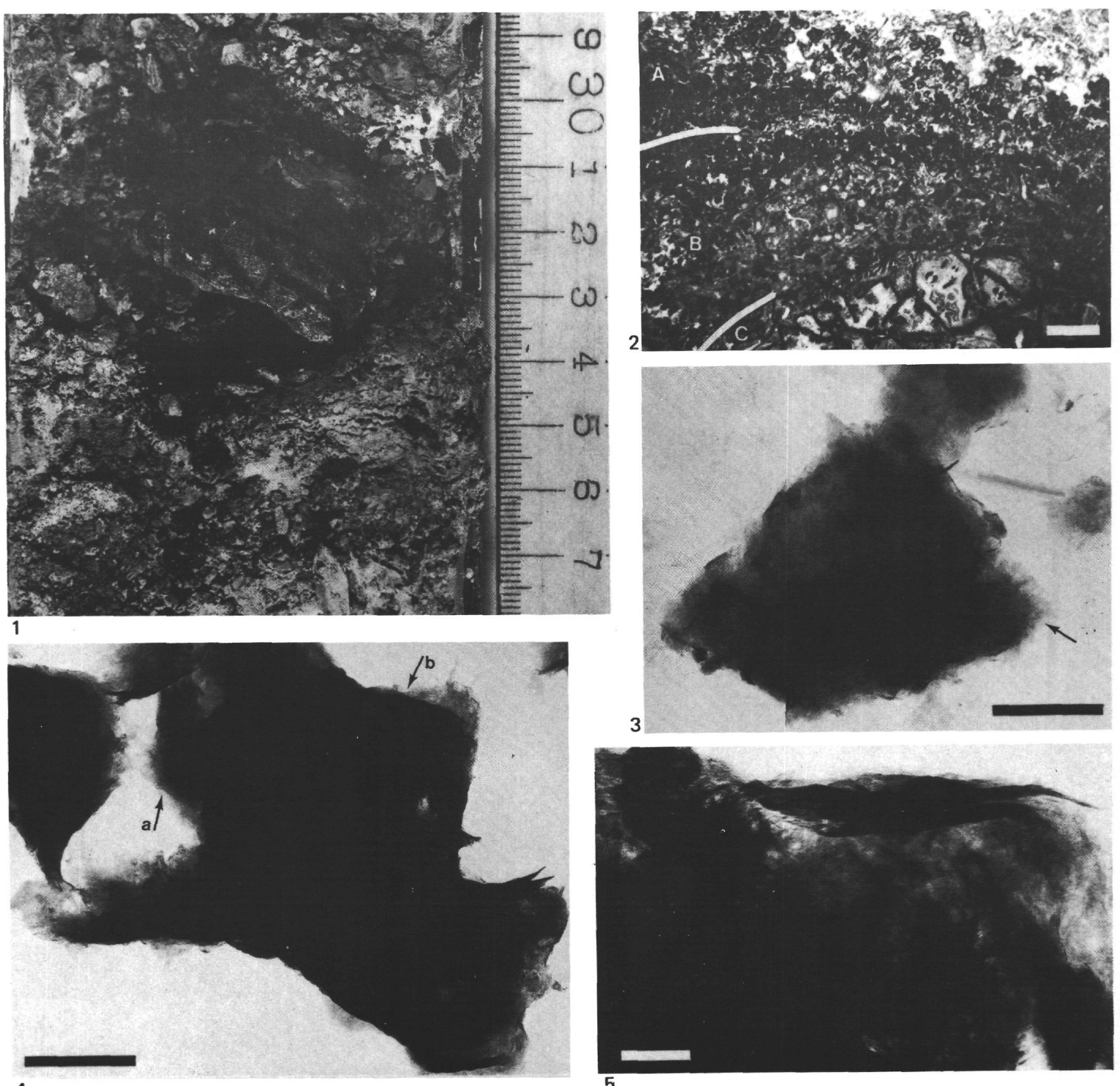

3

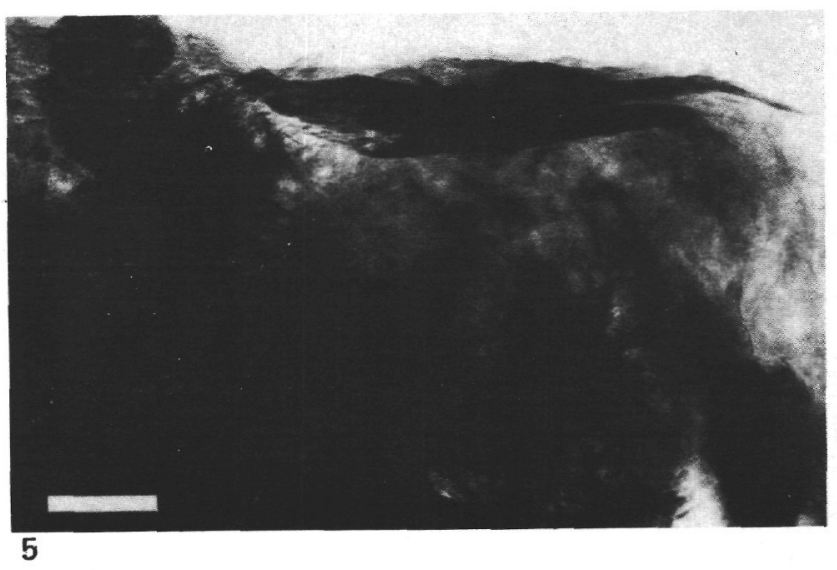

Plate 6. Crust fragment in Miocene marly nannofossil ooze from Site 520. 1. General view of sediment at 520-15-2, 29-37 cm. Siliceous and iron-rich crust appear within the marly calcareous ooze. 2. Polished section of the crust, revealing three main layers of oxides and silicates. From top to bottom: A and B, successive fine deposits of Fe-oxide and clays, with colloform structures as deep-sea metalliferous nodules; layer $\mathrm{C}$ is a hyaloclastite comprising plagioclases and scarce pyroxene, both of which are altered and coated by yellow to red clays and iron oxides. Scale bar $=1 \mathrm{~mm}$. 3. TEM microphotographs of the clay fraction from marly nannofossil ooze below the iron crust $(520-15-2,38-40 \mathrm{~cm})$. Smectite particle with light curled edges and lath-shaped overgrowths (at arrow). Scale bar $=0.5 \mu \mathrm{m}$. 4. General view of large smectites with curled outlines fringed in places with fine laths (a) or bundles of fibers (b); compare with Plate 5, fig. 4. Scale bar $=0.5 \mu \mathrm{m}$. 5. Detail of the bundles of bent fibers (b), which are similar to authigenic iron smectites growing over the large primary smectites. Scale bar $=0.1 \mu \mathrm{m}$. 